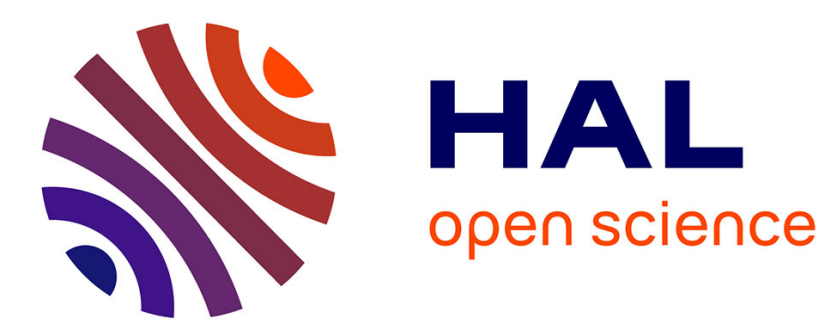

\title{
Influence d'un état désaimanté anisotrope sur l'aimantation dans le domaine de Rayleigh
}

R. Vergne, J.L. Porteseil, Z. Blazek

\section{To cite this version:}

R. Vergne, J.L. Porteseil, Z. Blazek. Influence d'un état désaimanté anisotrope sur l'aimantation dans le domaine de Rayleigh. Revue de Physique Appliquée, 1975, 10 (4), pp.195-211. 10.1051/rphysap:01975001004019500 . jpa-00243905

\section{HAL Id: jpa-00243905 https://hal.science/jpa-00243905}

Submitted on 1 Jan 1975

HAL is a multi-disciplinary open access archive for the deposit and dissemination of scientific research documents, whether they are published or not. The documents may come from teaching and research institutions in France or abroad, or from public or private research centers.
L'archive ouverte pluridisciplinaire HAL, est destinée au dépôt et à la diffusion de documents scientifiques de niveau recherche, publiés ou non, émanant des établissements d'enseignement et de recherche français ou étrangers, des laboratoires publics ou privés. 


\title{
INFLUENCE D'UN ÉTAT DÉSAIMANTÉ ANISOTROPE SUR L'AIMANTATION DANS LE DOMAINE DE RAYLEIGH
}

\author{
R. VERGNE, J. L. PORTESEIL et Z. BLAZEK $\left({ }^{*}\right)$ \\ Laboratoire de Magnétisme du C. N. R. S., B. P. 166, Centre de Tri, \\ 38042 Grenoble Cedex, France
}

(Reçu le 17 juin 1974, révisé le 3 février 1975, accepté le 5 mars 1975)

\begin{abstract}
Résumé. - La désaimantation d'un échantillon polycristallin par un champ alternatif évanescent conduit à un état de référence anisotrope si la substance possède plusieurs axes de facile aimantation. Les auteurs étudient l'influence de cette anisotropie de la désaimantation sur l'aimantation dans le domaine de Rayleigh d'une substance polycristalline de structure cubique ayant, comme le fer, une forte anisotropie et 3 axes de facile aimantation suivant les axes quaternaires du cristal. Les coefficients de la loi Rayleigh macroscopique dépendent du type de désaimantation utilisée. Le modèle proposé interprète ces résultats par l'évolution de plusieurs populations de parois de Bloch en fonction de l'amplitude maximale du champ de désaimantation. Les résultats expérimentaux obtenus à partir des courbes de première aimantation d'un acier s'interprètent bien dans le cadre de ce modèle et confirment de façon satisfaisante les hypothèses faites sur les mécanismes de désaimantation.
\end{abstract}

\begin{abstract}
Demagnetizing a polycristalline sample by an alternating field leads to an anisotropic reference state if the material has several easy axes. The authors study the effect of anisotropy of demagnetization on the virgin magnetization of a polycristalline cubic material in the Rayleigh region, assuming that the sample has a strong magnetocrystalline anisotropy and three axes along the four-fold axes of the crystal (iron). The coefficients of the macroscopic Rayleigh law depend on the previous demagnetization. A model is suggested which interprets thése results in terms of several types of Bloch walls, the percentages of which depend on the peak amplitude of the demagnetizing field. The experimental results relating to the magnetization curves of a steel sample are consistent with the model and give satisfactory confirmation of the assumptions made.
\end{abstract}

1. Introduction. - 1.1 LES LOIS DE RAYLEIGH. On sait depuis longtemps qu'un grand nombre de substances magnétiques suivent, après avoir été désaimantées dans un champ alternatif, les lois découvertes expérimentalement par Lord Rayleigh [1].

Lorsque le champ magnétique a atteint, par valeurs décroissantes, une valeur $H_{1}$, l'aimantation ayant dans ces conditions une valeur $J_{1}$ et que l'on fait croître $H$ à partir de $H_{1}$, l'aimantation $J$ s'écrit :

$$
J-J_{1}=a\left(H-H_{1}\right)+\frac{b}{2}\left(H-H_{1}\right)^{2} \quad H>H_{1} .
$$

De même, lorsque le champ magnétique a atteint par valeurs croissantes une valeur $\mathrm{H}_{2}$ et l'aimantation une valeur $J_{2}$, lorsqu'on fait décroître le champ, l'aimantation $J$ s'écrit algébriquement :

(*) National Research Institute for Materials, S. V. U. M., Prague 1, Opletalova 25, Czechoslovakia.

$$
J-J_{2}=a\left(H-H_{2}\right)-\frac{b}{2}\left(H-H_{2}\right)^{2} \quad H<H_{2} .
$$

Ces lois conduisent pour la courbe de première aimantation à une variation parabolique de l'aimantation en fonction du champ magnétisant :

$$
J=a H+b H^{2} .
$$

Dans ces expressions, $a$ est la susceptibilité initiale et $b$ la constante de Rayleigh. Les deux premières relations permettent de décrire le cycle d'hystérésis et les propriétés de la substance au voisinage d'un point d'aimantation déterminé $\left(J_{0}, H_{0}\right)$.

Le domaine de champ où ces lois sont applicables est relativement restreint : sa limite supérieure est de l'ordre du dixième du champ coercitif. Certains auteurs ont montré $[2,3]$ et nous avons vérifié que ces lois ne sont plus valables dans les champs très faibles. L. Néel [4] a donné une interprétation théorique de ces 
lois qui repose sur l'analyse précise des déplacements d'une paroi de Bloch dans un milieu irrégulièrement perturbé. G. Bonnet [5] a justifié et complété ce modèle en appliquant la théorie des fonctions aléatoires aux déplacements de parois. Dans une première partie de son mémoire, L. Néel montre qu'une paroi isolée obéit à une loi du type Rayleigh. Dans la seconde partie, il applique les résultats obtenus à une substance réelle à domaines multiples. Il traite complètement le cas d'un polycristal parfait d'une substance magnétiquement uniaxe et donne, dans ce cas, l'expression des constantes macroscopiques en fonction des paramètres introduits. Il esquisse également le calcul dans le cas d'une substance cubique, mais il considère toujours un état désaimanté isotrope. Or, si cette hypothèse est tout à fait justifiée dans le cas d'une substance uniaxe, il n'en est plus de même pour une substance polycristalline de structure cubique ayant plusieurs axes de facile aimantation.

1.2 L'ANISOTROPIE DE LA DÉSAIMANTATION. Comme l'aimantation d'une substance ferromagnétique dépend non seulement de la valeur actuelle du champ magnétisant mais également de toute son histoire magnétique, il est nécessaire de savoir réaliser un état de référence reproductible d'aimantation macroscopique nulle. Parmi les moyens dont on dispose pour obtenir un état désaimanté, nous en envisagerons deux : la désaimantation thermique et l'action d'un champ alternatif évanescent. La première est obtenue en réchauffant l'échantillon au-dessus de sa température de Curie et en le refroidissant en champ nul. Elle est rarement utilisée car elle n'est pas facile à mettre en œuvre et présente des inconvénients si la température de Curie est élevée. Elle peut alors entraîner des modifications de la structure et des propriétés magnétiques de l'échantillon. La désaimantation thermique d'un polycristal parfait conduit à un état désaimanté isotrope. En effet, en détruisant l'ordre magnétique, l'agitation thermique ne privilégie aucune direction de facile aimantation et après refroidissement en champ nul toutes ces directions sont également occupées par les vecteurs $\mathbf{J}_{\mathrm{s}}$. Il en résulte, si le polycristal est parfait, un état désaimanté isotrope. Il n'en est plus de même de la désaimantation alternative si la substance possède, comme le fer, plusieurs axes de facile aimantation. En effet, dans un cristallite, au cours de la désaimantation alternative, les directions de facile aimantation ne sont plus énergétiquement équivalentes; la direction la plus proche du champ de désaimantation est privilégiée et ceci d'autant plus que l'amplitude maximale de ce champ est grande. Au cours de la désaimantation les vecteurs $\mathbf{J}_{\mathrm{s}}$ auront tendance à se placer suivant les directions de facile aimantation énergétiquement les plus favorables. Il en résulte une répartition anisotrope des vecteurs $\mathbf{J}_{\mathrm{s}}$ dans l'état désaimanté et une structure en domaines qui reflète cette anisotropie. Ce phénomène n'existe plus dans une substance uniaxiale, car au niveau du cristallite il n'y a plus de choix possible, une seule direction étant privilégiée. Dans un polycristal réel, l'existence de défauts fait que dans certaines régions les axes de facile aimantation ne sont plus énergétiquement équivalents. On a un caractère uniaxial local très prononcé entraînant dans ces régions une configuration en domaines déterminée qui ne dépend plus du champ de désaimantation. Si ces régions sont réparties au hasard dans le polycristal, il en résulte pour l'ensemble de celles-ci un état désaimanté isotrope par compensation. Dans un tel matériau une partie seulement sera sensible au phénomène d'anisotropie de la désaimantation.

Avant d'envisager les conséquences qu'entraîne l'existence de l'anisotropie de la désaimantation sur l'aimantation des substances ferromagnétiques dans les champs faibles, nous allons faire un bref historique des travaux consacrés à ces questions.

1.3 TravauX CONSACRÉS A LA DÉSAIMANTATION ET A L'ANISOTROPIE DE LA DÉSAIMANTATION. - Nous citerons en premier lieu un certain nombre d'études consacrées à la différence entre la désaimantation thermique et la désaimantation alternative faite dans la direction du champ de mesure : [6] à [11]. Ces études ont porté sur le fer ou sur les matériaux à base de fer, les échantillons étaient soit des polycristaux, soit des monocristaux. Les auteurs trouvent en général que la substance s'aimante moins facilement après une désaimantation thermique. Ils expliquent cette différence [12] comme étant une conséquence des deux mécanismes que nous avons développés au paragraphe précédent. Signalons cependant que d'autres auteurs $[13,14]$ ont trouvé pour le nickel le résultat contraire, soit $\chi_{\mathrm{T}}>\chi_{\mathrm{A}}$. On a aussi observé une situation plus complexe sur le ferrite de $\mathrm{Mn}-\mathrm{Zn}$ : une désaimantation alternative effectuée à la température ambiante conduit à $\chi_{\mathrm{T}}<\chi_{\mathrm{A}}$, alors que la même désaimantation effectuée au voisinage de la température de Curie conduit au résultat inverse.

Les travaux où l'on étudie des désaimantations alternatives faites dans des directions autres que la direction de mesure sont moins nombreux. La majeure partie a pour origine le modèle d'hystérésis à plusieurs dimensions proposé par L. Néel [16] en 1959. Les auteurs [17, 18, 19, 20, 21] montrent que l'anisotropie de la désaimantation que l'on obtient sur diverses substances polycristallines est liée à l'existence de plusieurs axes de facile aimantation dans chaque cristallite comme nous l'avons précisé au paragraphe précédent. Cet effet n'existe pas dans le cobalt hexagonal polycristallin qui a un seul axe de facile aimantation, mais réapparaît quand on traite l'échantillon de manière à ce qu'une partie du cobalt soit en phase cubique, où il possède alors plusieurs axes de facile aimantation [22]. L'anisotropie de la désaimantation, observée sur un polycristal cubique, s'atténue et disparaît quand on introduit progressivement le caractère uniaxial, par exemple en appliquant une contrainte uniaxiale au matériau $[17,18,20]$. Ces auteurs ont également 
montré que l'aimantation de la substance dans un champ donné est maximale dans la direction de désaimantation [17] à [22]. Une étude relative à un acier au silicium laminé [23] conduit à la même conclusion. Les ferrites ont été également étudiées [24, 25]. Ce dernier travail [25] est très complet mais il a été fait en dehors du domaine de Rayleigh.

Il ressort de cet exposé que nous ne disposons pas d'un ensemble de résultats expérimentaux suffisamment détaillés et précis qui pourraient servir de base à une interprétation du phénomène. Ceci nous a incités à entreprendre cette étude. Pour interpréter nos résultats nous avons été conduits d'une part à développer un modèle d'anisotropie de la désaimantation, d'autre part à calculer l'aimantation dans le domaine de Rayleigh d'un polycristal d'une substance magnétique ayant plusieurs axes de facile aimantation. Nous nous sommes bornés au cas $\mathrm{du}$ fer et nous avons fait le calcul dans le cadre du modèle de Néel relatif aux lois de Rayleigh [4].

2. Expressions théoriques. - Nous avons développé dans la référence [26] un modèle d'anisotropie de la désaimantation. Il nous paraît indispensable pour comprendre la suite de l'exposé d'en rappeler les grandes lignes.

2.1 ElABORATION D'UN MODÈlE D'ANISOTROPIE DE LA DÉSAIMANTATION. EXPRESSION DE L'AIMANTATION DANS LE DOMAINE DE RAYLEIGH DANS LE CAS PARTICULIER D'UN MODÈle PLAN. DÉFINITION D'UN ÉTAT DE RÉFÉRENCE ISOTROPE. - Pour élaborer un tel modèle, nous sommes amenés à faire des hypothèses relatives au polycristal, aux mécanismes de désaimantation et aux mécanismes d'aimantation dans le domaine de Rayleigh.

En ce qui concerne le polycristal, nous supposerons que les axes des cristallites sont répartis au hasard. Nous supposerons également qu'il existe dans celui-ci deux types de régions élémentaires: dans l'une de celles-ci (I) la structure en domaines élémentaires dans l'état désaimanté dépend du mode de désaimantation ; dans l'autre (II) il n'en est rien, aux centres de nucléation près. Cette répartition est alors statistiquement entièrement déterminée au cours de la désaimantation par les conditions locales imposées par la structure du polycristal. Elle ne dépend pas du mode de désaimantation, à l'exception des centres de nucléation que l'on peut négliger quand on envisage la structure en domaines de l'ensemble des régions (II). Par contre c'est l'évolution des centres de nucléation au cours de la désaimantation qui conditionne la structure en domaines des régions (I). Pour la commodité de l'exposé, nous appellerons phases I et II l'ensemble des régions élémentaires du type I et II. Nous associons à la phase I une répartition anisotrope des polarisations $\mathbf{P}\left({ }^{1}\right)$. Nous supposons que dans la phase II

(1) Remarque. - Le vecteur polarisation $\mathbf{P}$ relatif à une paroi de Bloch séparant deux domaines élémentaires aimantés respec- aucune direction de polarisation n'est privilégiée en moyenne. Il en résulte une répartition isotrope des polarisations.

A. Nous allons montrer que l'application à la phase I d'un champ alternatif évanescent de direction déterminée conduit à une répartition anisotrope des polarisations : même si cette répartition était initialement isotrope. Nous déterminerons ensuite cette répartition.

Nous allons d'abord préciser la répartition de l'ensemble des directions de facile aimantation du polycristal en fonction de l'angle qu'elles font avec la direction du champ de désaimantation interne qui lui est appliqué. Dans la suite de cet exposé nous supposerons toujours que l'échantillon est limité par une surface du second degré. Le champ $\mathfrak{H}$ dans un cristallite est la somme vectorielle de trois termes : le champ appliqué, le champ démagnétisant de forme et le champ de dispersion dû aux charges magnétiques libres dans le cristal et aux interactions entre domaines. Dans ce qui suit, nous négligerons ce dernier. Considérons la situation dans un cristallite. Soit 1, 2, 3, les trois directions de facile aimantation. Ce sont ici les axes quaternaires du cristallite. Elles forment un trièdre trirectangle (Fig. 1).

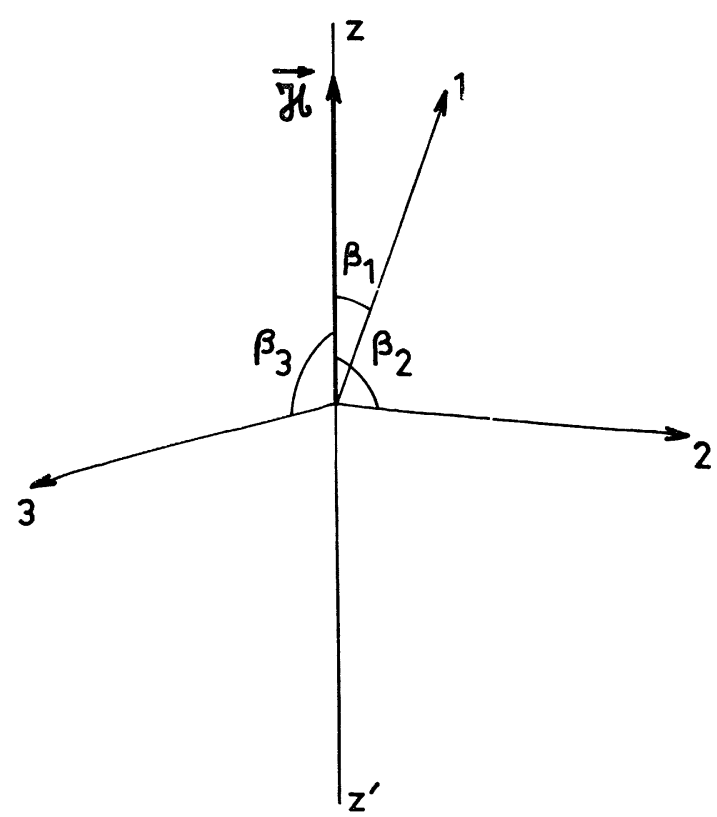

Fig. 1. - Les directions du champ de désaimantation $\mathscr{H}$ et des axes de facile aimantation $1,2,3$.

Soit $\beta_{1}, \beta_{2}, \beta_{3}$ les angles de ces directions avec la direction $Z Z^{\prime}$ du champ $\mathscr{H}$ de désaimantation. Posons $\beta_{1}<\beta_{2}<\beta_{3}$ et donnons au trièdre $1,2,3$ toutes les orientations possibles avec une égale probabilité. Nous pouvons calculer $P_{1}\left(\beta_{1}\right), P_{2}\left(\beta_{2}\right), P_{3}\left(\beta_{3}\right)$ les probabilités pour une direction de facile aimantation faisant un

tivement suivant $\mathbf{J}_{1}$ et $\mathbf{J}_{2}$ est par définition le vecteur $\mathbf{P}=\mathbf{J}_{\mathbf{2}}-\mathbf{J}_{\mathbf{1}}$. Le déplacement de la paroi affectant un volume $\Delta V$ entraîne une variation d'aimantation $P \Delta V$ dans la direction de $P$. 
angle $\beta$ avec la direction $\mathscr{H}$, d'être respectivement dans la situation 1, 2, 3, c'est-à-dire la plus proche, la moyennement proche ou la plus éloignée de $\beta$. Les $P_{i}\left(\beta_{i}\right)$ définissent la répartition des directions de facile aimantation dans le polycristal. La figure 2 donne l'allure de ces probabilités.

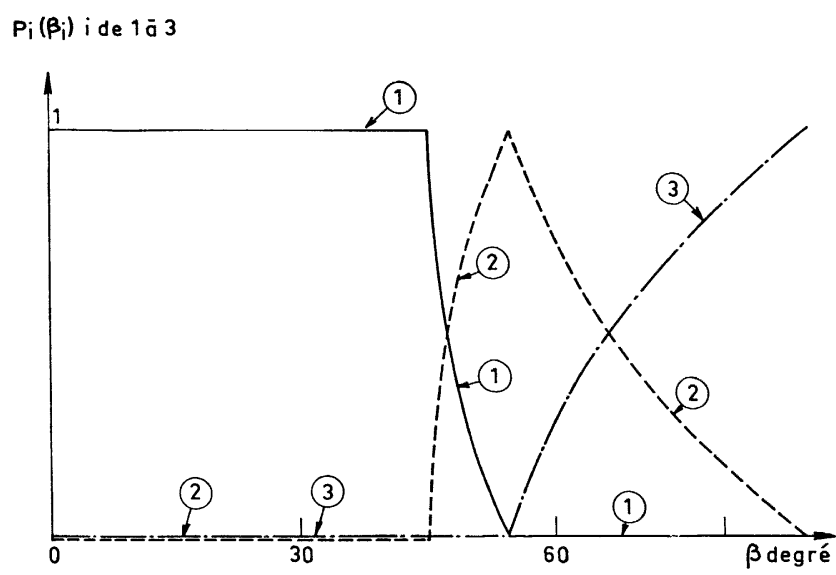

FIG. 2. - La répartition des directions de facile aimantation.

Dans un cristal de type fer, on a deux sortes de parois de Bloch : des parois à $180^{\circ}$ et des parois à $90^{\circ}$. Pour former ces parois il faut associer deux vecteurs aimantation spontanée $\mathbf{J}_{\mathbf{s}}$ soit antiparallèlement, soit perpendiculairement, chacun de ces vecteurs étant suivant une direction de facile aimantation. L'ensemble des combinaisons se regroupe en 6 possibilités qui sont celles du tableau I, ce qui entraîne 6 types de vecteurs polarisation possibles.

\section{TABLEAU I}

$\begin{array}{llccc}\text { Parois à } 180^{\circ} & \text { Type } & 1-\overline{1} & 2-\overline{2} & 3-\overline{3} \\ & \text { Indice } & 1 & 2 & 3 \\ \text { Parois à } 90^{\circ} & \text { Type } & 1-2 & 1-3 & 2-3 \\ & \text { Indice } & 4 & 5 & 6\end{array}$

Pour former une paroi à $180^{\circ}$ du type $1-\overline{1}$ on associe antiparallèlement 2 vecteurs $\mathbf{J}_{\mathrm{s}}$ placés suivant 2 directions de facile aimantation dans la situation 1 , c'est-àdire les plus proches de $Z Z^{\prime}$. Alors que pour former une paroi à $90^{\circ}$ du type 1-2 on associe perpendiculairement 2 vecteurs $\mathbf{J}_{\mathrm{s}}$ placés suivant deux directions de facile aimantation, étant respectivement dans la situation 1 et 2 . Nous noterons ces parois de 1 à 6 .

Il faut faire ici des hypothèses relatives aux mécanismes de désaimantation par un champ alternatif évanescent de direction déterminée. Nous poserons que la population de ces 6 types de parois dépend de l'amplitude maximale $\mathcal{H}_{\mathrm{m}}$ de $\mathscr{H}$. Pour $\mathcal{H}_{\mathrm{m}}$ tendant vers zéro il y a équipartition. Quand $\mathscr{H}_{\mathrm{m}}$ croît, les parois les plus défavorisées, 3 et 6 , qui sont formées à partir des vecteurs $\mathbf{J}_{\mathbf{s}}$ les plus éloignés de la direction de $\boldsymbol{J}$ disparaissent les premières, puis les parois 2 et 5 disparaissent à leur tour et il ne reste plus que les parois 1 et 4 . Pour une amplitude suffisante de $\mathcal{H}_{\mathrm{m}}$ les vecteurs $\mathbf{J}_{\mathrm{s}}$ se placent dans l'état désaimanté suivant la direction de facile aimantation la plus proche de celle du champ de désaimantation. C'est l'hypothèse généralement admise, sans justification d'ailleurs.

Mais ce qui nous intéresse ce n'est pas la répartition des vecteurs $\mathbf{J}_{\mathbf{s}}$ mais celle des 6 vecteurs polarisation possible. Nous les calculerons à partir des $P_{i}\left(\beta_{i}\right)$. On obtient finalement 6 répartitions anisotropes $P_{k}(\beta)$ ( $k$ de 1 à 6 ). La figure 3 donne les répartitions relatives aux parois à $90^{\circ}$. En ce qui concerne les parois à $180^{\circ}$ le calcul montre que les $P_{k}(\beta)$ ( $k$ de 1 à 3 ) sont identiques aux $P_{i}\left(\beta_{i}\right)$. La répartition des polarisations dans la phase I étant une combinaison linéaire de ces 6 répartitions anisotropes, sera elle aussi anisotrope.

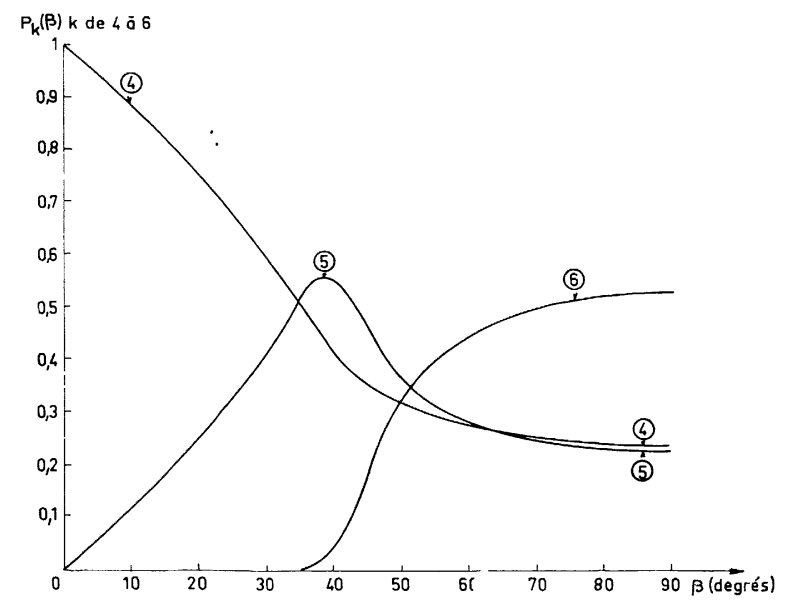

Fig. 3. - Les répartitions anisotropes relatives aux parois à $90^{\circ}$ ( $k$ de 4 à 6$)$; celles ayant trait aux parois à $180^{\circ}(k$ de 1 à 3$)$ sont analogues aux répartitions de la figure 2 .

B. Calcul de l'aimantation du polycristal dans le domaine de Rayleigh. - Nous supposerons que les variations d'aimantation sont exclusivement dues à des déplacements de parois, que ces déplacements sont indépendants et qu'au cours d'un tel processus les vecteurs $\mathbf{J}_{\mathrm{s}}$ gardent une direction fixe. Nous supposerons également qu'il n'apparaît pas de pôles libres dans le cristal et nous négligerons les effets de magnétostriction. Ces hypothèses sont celles que l'on admet généralement dans le domaine de Rayleigh [4]. Elles négligent, comme nous l'avons fait plus haut, l'existence d'un champ de dispersion. De récents travaux de J. L. Porteseil consacrés aux mécanismes d'aimantation en champs faibles dans des structures en domaines simples ont montré de manière directe l'existence d'interactions entre parois. Ces interactions magnétostatiques sont d'autant plus importantes qu'il y a peu de domaines en présence et se moyennent très vite quand leur nombre croît. Il nous paraît raisonnable de les négliger dans une structure complexe comportant de très nombreux domaines, du moins en ce qui concerne la première aimantation du polycristal.

Une structure en domaines élémentaires donnée est alors équivalente à une répartition de vecteurs polarisation, chacun de ces vecteurs étant affecté à un 
paroi [16]. Dans son mémoire [4] L. Néel montre qu'une paroi isolée suit une loi d'aimantation du type Rayleigh. J. L. Porteseil a donné une vérification expérimentale directe en étudiant l'aimantation d'un cadre monocristallin de $\mathrm{Fe}-\mathrm{Si}$ comportant une seule paroi à $180^{\circ}$ mobile. Aussi écrirons-nous que suivant une direction de polarisation la variation élémentaire d'aimantation est de la forme :

$$
\delta \mathfrak{J}=a h_{\mathrm{e}}+b h_{\mathrm{e}}^{2}
$$

où $h_{\mathrm{e}}$ est la projection du champ interne $\mathbf{H}$ suivant le vecteur polarisation $\mathbf{P}$ et $a$ et $b$ deux constantes élémentaires spécifiques du type de paroi considéré. Pour la phase I nous utiliserons 2 jeux de constantes : $a_{1}, b_{1}$, et $a_{2}, b_{2}$ ayant trait respectivement aux parois à $180^{\circ}$ et $90^{\circ}$. En ce qui concerne la phase II, comme nous envisageons une répartition isotrope des polarisations, nous ne différencions plus les deux types de parois et nous considérons une paroi moyenne de constantes $a$ et $b$ (cf. Tableau II). L'aimantation relative à un vecteur $\mathbf{P}$ dans une direction $\mathbf{H}$ s'obtient en projetant $\delta J$ sur $H$. L'aimantation globale est la somme de toutes les aimantations élémentaires compte tenu de la répartition des polarisations. On suppose de plus que la surface totale des parois reste constante. Cette hypothèse a été discutée et justifiée par L. Néel dans [4]. On calcule ainsi la loi relative à chaque type de paroi à partir de la répartition des polarisations correspondantes. On a dans le cas le plus général 7 lois. Pour la phase I du polycristal on a une combinaison linéaire des 6 lois correspondant aux six types de parois. Pour la phase II on a une loi correspondant à une répartition isotrope. Pour avoir l'aimantation du polycristal, on introduit les proportions $\varepsilon$ et $(1-\varepsilon)$ des phases I et II. On écrit ensuite qu'elle est la somme des aimantations des deux phases. Pour des amplitudes maximales $\mathfrak{H}_{\mathrm{m}}$ du champ de désaimantation inférieures à quelques fois le champ coercitif $\left(\sim 3 H_{\mathrm{c}}\right)$ il faut considérer les 7 types de parois. Toutefois, le problème se simplifie quand l'amplitude $\mathscr{H}_{\mathrm{m}}$ est suffisante $\left(>3 H_{\mathrm{c}}\right)$; il ne subsiste alors que 3 types de parois.

Le tableau II résume le modèle et donne l'expression générale de la loi relative à la courbe de première aimantation de la substance. Les coefficients de la loi de Rayleigh macroscopique sont des combinaisons linéaires des coefficients des lois de Rayleigh ayant trait à chaque type de paroi. Dans la suite de l'exposé, nous nous bornerons à un problème plan. L'échantillon est constitué par un ellipsoïde aplati d'axe de révolution $O Y$ (cf. Fig. 4) ; le champ magnétique appliqué, la direction de mesure et le champ de désaimantation sont dans le plan $Z O X$. L'aimantation dans le domaine de Rayleigh s'écrit :

$$
\jmath_{H}^{\gamma}=A^{\gamma} H+B^{\gamma} H^{2}
$$

$\jmath_{H}^{\gamma}$ désigne l'aimantation prise par la substance suivant la direction du champ magnétisant $\mathbf{H}$, après avoir été désaimantée par un champ alternatif évanescent $\boldsymbol{H} \boldsymbol{U}$ de

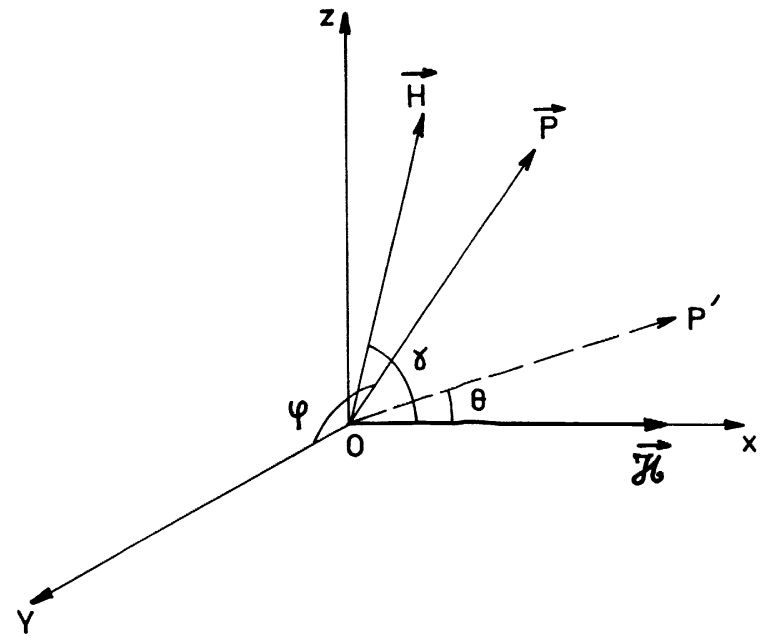

Fig. 4. - Les directions du champ appliqué, de mesure et du champ de désaimantation dans le plan $Z O X$.

direction $\gamma$ (cf. Fig. 4). Les constantes $A^{\gamma}$ et $B^{\gamma}$ macroscopiques correspondantes ont pour expression :

$$
\begin{aligned}
& A^{\gamma}=\sum_{k=1}^{3}\left(a_{1}^{\prime}\right)_{k} Q_{k \mathrm{r}}^{\gamma}+\sum_{k=4}^{6}\left(a_{2}^{\prime}\right)_{k} Q_{k \mathrm{r}}^{\gamma}+\frac{1}{3} a^{\prime} \\
& B^{\gamma}=\sum_{k=1}^{3}\left(b_{1}^{\prime}\right)_{k} Q_{k \mathrm{i}}^{\gamma}+\sum_{k=4}^{6}\left(b_{2}^{\prime}\right)_{k} Q_{k \mathrm{i}}^{\gamma}+\frac{1}{4} b^{\prime} .
\end{aligned}
$$

Les quantités $\left(a_{1}^{\prime}\right)_{k} \ldots a^{\prime},\left(b_{1}^{\prime}\right)_{k} \ldots b^{\prime}$ sont définies dans le tableau II. Les sommations sur $k$ de 1 à 6 correspondent aux 6 types de parois définis dans le tableau I. Les quantités $Q_{k \mathrm{r}}^{\gamma}$ et $Q_{k \mathrm{i}}^{\gamma}$ ont trait respectivement aux parties réversibles et irréversibles de l'aimantation. Ce sont des facteurs géométriques, spécifiques de chaque type de paroi, qui sont des fonctions de $\gamma$ et de la répartition $P_{k}(\beta)$ des vecteurs polarisation correspondant au type de paroi $k$ considéré. Ces quantités sont calculables dans le cadre du modèle et leurs valeurs sont données en fonction de $\gamma$ par les figures 5 et 6 . Il est possible de déterminer expérimentalement l'ensemble des coefficients $\left(a_{1}^{\prime}\right)_{k} \ldots a^{\prime},\left(b_{1}^{\prime}\right)_{k} \ldots b^{\prime}$ par des mesures de susceptibilité (cf. [26]). La connaissance de ces coeffi-

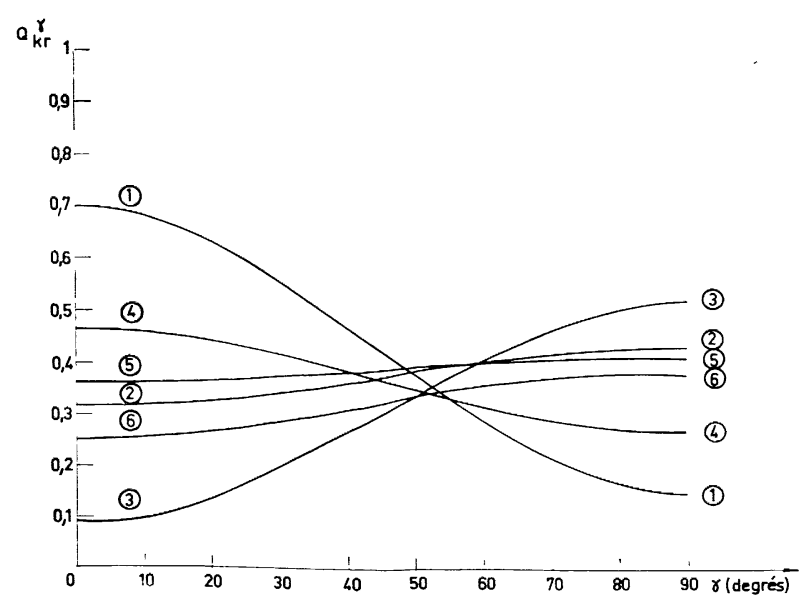

Fig. 5. $-Q_{k \mathrm{r}}^{\gamma}$ en fonction de $\gamma$ pour $k=1$ à 6 . 


\section{TABleAU II}

Paramètres élémentaires

Phase I

Proportion dans le cristal

$\varepsilon$

Parois à $180^{\circ}: a_{1}, b_{1}$ Parois à $90^{\circ}: a_{2}, b_{2}$

Aimantation macroscopique du polycristal

Phase I

Type de paroi :

$180^{\circ}: 1,2,3$

$90^{\circ}: 4,5,6$

Population :

$$
\begin{aligned}
& 180^{\circ}: P_{k} k \text { de } 1 \text { à } 3 \\
& 90^{\circ}: P_{k} k \text { de } 4 \text { à } 6\left(\sum_{k} P_{k}=1\right)
\end{aligned}
$$

Nombre de parois :

$180^{\circ}: \varepsilon P_{k} k$ de 1 à 3

$90^{\circ}: \varepsilon P_{k} k$ de 4 à 6

Combinaison des constantes élémentaires intervenant dans la loi macroscopique, relatives à chaque type de paroi :

$$
\begin{aligned}
& 180^{\circ}:\left(a_{1}^{\prime}\right)_{k}=a_{1} \varepsilon P_{k} k \text { de } 1 \text { à } 3 \\
& \left(b_{1}^{\prime}\right)_{k}=b_{1} \varepsilon P_{k} \\
& 90^{\circ}:\left(a_{2}^{\prime}\right)_{k}=a_{2} \varepsilon P_{k} k \text { de } 4 \text { à } 6 \\
& \left(b_{2}^{\prime}\right)_{k}=b_{2} \varepsilon P_{k}
\end{aligned}
$$

Phase II

Proportion dans le cristal

$1-\varepsilon$

Parois moyennes $a, b$

\section{Phase II}

Type de paroi :

Moyenne

Nombre de parois :

Moyenne $(1-\varepsilon)$.

Combinaison des constantes élémentaires intervenant dans la loi macroscopique, relatives à chaque type de paroi :

Cas particulier d'un problème plan (cf. Fig. 1).

Loi d'aimantation élémentaire : courbe de première aimantation

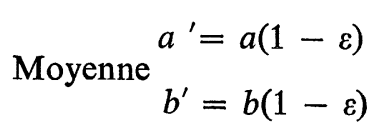

Phase I

$$
\begin{array}{rll}
180^{\circ}: 1,2,3 & (\mathfrak{J})_{k}=\left(a_{1}^{\prime}\right)_{k} Q_{k \mathrm{r}}^{\gamma} H+\left(b_{1}^{\prime}\right)_{k} Q_{k \mathrm{i}}^{\gamma} H^{2} & k \text { de } 1-3 \\
90^{\circ}: 4,5,6 & (\mathfrak{J})_{k}=\left(a_{2}^{\prime}\right)_{k} Q_{k \mathrm{r}}^{\gamma} H+\left(b_{2}^{\prime}\right)_{k} Q_{k \mathrm{i}}^{\gamma} H^{2} & k \text { de } 4-6 .
\end{array}
$$

Phases II

Moyenne : $=a^{\prime} \frac{1}{3} H+b^{\prime} \frac{1}{4} H^{2}$

Loi d'aimantation totale : courbe de première aimantation

Phase I + II

$$
\begin{aligned}
& J_{H}^{\gamma}=\left\{\sum_{k=1}^{3}\left(a_{1}^{\prime}\right)_{k} Q_{k \mathrm{r}}^{\gamma}+\sum_{k=4}^{6}\left(a_{2}^{\prime}\right)_{k} Q_{k \mathrm{r}}^{\gamma}+a^{\prime} \frac{1}{3}\right\} H+\left\{\sum_{k=1}^{3}\left(b_{1}^{\prime}\right)_{k} Q_{k \mathrm{i}}^{\gamma}+\sum_{k=4}^{6}\left(b_{2}^{\prime}\right)_{k} Q_{k \mathrm{i}}^{\gamma}+b^{\prime} \frac{1}{4}\right\} H^{2} \\
& J_{H}^{\gamma}=A^{\gamma} H+B^{\gamma} H^{2} .
\end{aligned}
$$




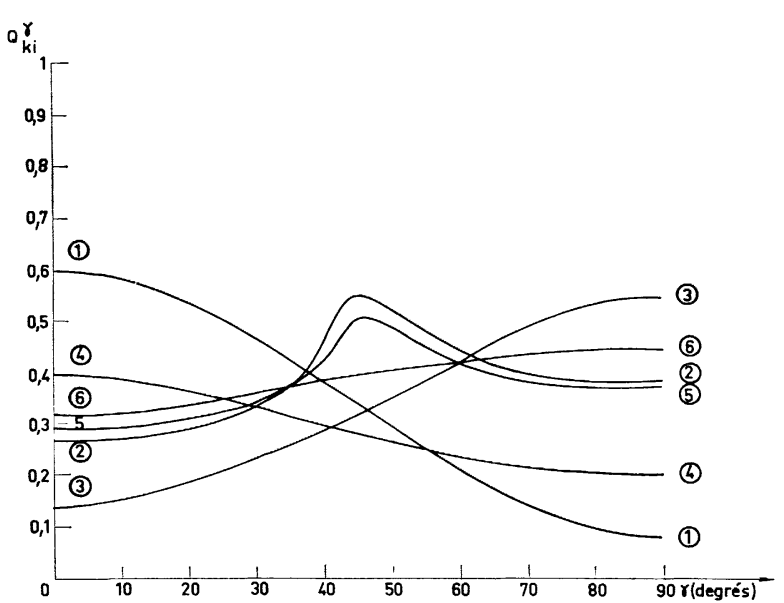

FIG. 6. $-Q_{k \mathrm{i}}^{\gamma}$ en fonction de $\gamma$ pour $k=1$ à 6 .

cients permet d'analyser l'aimantation globale en termes d'aimantation dus à chaque type de paroi et caractérise complètement l'anisotropie de la désaimantation dans le cadre du modèle.

2.1.1 Définition d'un état de référence isotrope. - Pour apprécier l'importance de l'anisotropie de la désaimantation nous devons être en mesure de définir un état désaimanté de référence isotrope dans le plan. C'est le but de la désaimantation tournante que nous réalisons en appliquant à l'échantillon un champ alternatif évanescent en même temps que nous le faisons tourner à vitesse angulaire constante autour de son axe de révolution $O Y$. Nous obtenons par ce procédé un état moyen isotrope. En effet, l'anisotropie de la désaimantation de la phase I est moyennée et la phase II est isotrope quelle que soit la désaimantation. Les constantes $Q_{k \mathrm{r}}^{\gamma}$ et $Q_{k \mathrm{i}}^{\gamma}$ ( $k$ de 1 à 6 ) prennent leur valeur moyenne par rapport à $\gamma$. Ces valeurs sont données dans le tableau III. Les valeurs des constantes macroscopiques $A^{t}$ et $B^{t}$ de $A^{\gamma}$ et $B^{\gamma}$ en découlent.

\section{TABleau III}

\begin{tabular}{lllllll}
\multicolumn{4}{c}{ Parois à $180^{\circ}$} & \multicolumn{3}{c}{ Parois à $90^{\circ}$} \\
$k$ & 1 & 2 & 3 & 4 & 5 & 6 \\
$Q_{k \mathrm{r}}^{t}$ & 0,4252 & 0,3237 & 0,2509 & 0,3664 & 0,3501 & 0,2834 \\
$Q_{k \mathbf{i}}^{t}$ & 0,3367 & 0,3551 & 0,3081 & 0,2843 & 0,3501 & 0,3655
\end{tabular}

2.2 DÉSAIMANTATION DE GRANDE AMPLITUDE $\mathscr{H}_{\mathrm{m}}>3 H_{\mathrm{c}}$ - 2.2.1 Expression de la loi d'aimantation du polycristal. — L'échantillon est soumis à une désaimantation uniaxiale d'amplitude $\mathfrak{H}_{\mathrm{m}}>3 H_{\mathrm{c}}$. Seules subsistent alors dans la phase I les parois du type 1 et $4, P_{2}=P_{3}=P_{5}=P_{6}=0$ (cf. Tableau II). Si $P_{1}=(1-k)$ et $P_{4}=k$ sont leurs proportions respectives, nous aurons dans le polycristal réel $\varepsilon(1-k)$ parois à $180^{\circ}$ de type $\mathrm{I}, \varepsilon k$ parois à $90^{\circ} \mathrm{du}$ type 4 et $(1-\varepsilon)$ parois ne participant pas à l'anisotropie de la désaimantation.

La loi de Rayleigh macroscopique est une combinaison linéaire de 3 lois de Rayleigh élémentaires, relatives à chaque type de paroi. L'aimantation globale suivant le champ magnétisant $H$ s'écrit alors :

$$
\left\{\begin{array}{l}
J_{H}^{\gamma}=A^{\gamma} H+B^{\gamma} H^{2} \\
A^{\gamma}=a^{\prime} \frac{1}{3}+a_{1}^{\prime} Q_{1 \mathrm{r}}^{\gamma}+a_{2}^{\prime} Q_{4 \mathrm{r}}^{\gamma} \\
B^{\gamma}=b^{\prime} \frac{1}{4}+b_{1}^{\prime} Q_{1 \mathrm{i}}^{\gamma}+b_{2}^{\prime} Q_{4 \mathrm{i}}^{\gamma}
\end{array}\right.
$$

en posant

$$
\begin{aligned}
& \left(a_{1}^{\prime}\right)_{1}=a_{1}^{\prime} ; \quad\left(a_{2}^{\prime}\right)_{4}=a_{2}^{\prime} ; \\
& \left(b_{1}^{\prime}\right)_{1}=b_{1}^{\prime} \quad \text { et } \quad\left(b_{2}^{\prime}\right)_{4}=b_{2}^{\prime} .
\end{aligned}
$$

Seules les quantités $A^{\gamma}$ et $B^{\gamma}$ qui sont des combinaisons linéaires de $a_{1}^{\prime}, b_{1}^{\prime} ; a_{2}^{\prime}, b_{2}^{\prime} ; a^{\prime}, b^{\prime}$ sont directement accessibles à l'expérience.

2.2.2 Définition d'un état de référence du polycristal, cet état étant magnétiquement isotrope dans le plan ZOX. - Pour cela nous appliquons à l'échantillon une désaimantation tournante d'amplitude maximale $\mathfrak{H}_{\mathrm{m}}>3 H_{\mathrm{c}}$. L'aimantation correspondante a pour expression :

$$
\text { - }\left\{\begin{array}{l}
J_{H}^{t}=A^{t} H+B^{t} H^{2} \\
A^{t}=a_{1}^{\prime} Q_{1 \mathrm{r}}^{t}+a_{2}^{\prime} Q_{4 \mathrm{r}}^{t}+a^{\prime} \frac{1}{3} \\
B^{t}=b_{1}^{\prime} Q_{1 \mathrm{i}}^{t}+b_{2}^{\prime} Q_{4 \mathrm{i}}^{t}+b^{\prime} \frac{1}{4} .
\end{array}\right.
$$

2.2.3 Détermination des constantes élémentaires introduites à partir de la mesure des coefficients macroscopiques $A^{\gamma}, B^{\gamma}, A^{t}, B^{t}$ des lois de Rayleigh du polycristal. - Les quantités $Q$ sont des fonctions de $\gamma$ ayant la forme d'un produit de convolution ; nous les développerons en série de Fourier. Le calcul donne les résultats suivants :

$$
\begin{aligned}
& \left\{\begin{array}{l}
Q_{1 \mathrm{r}}^{\gamma}=A_{01}+A_{21} \cos 2 \gamma \\
Q_{4 \mathrm{r}}^{\gamma}=A_{04}+A_{24} \cos 2 \gamma
\end{array}\right. \\
& \left\{\begin{array}{l}
Q_{1 \mathrm{i}}^{\gamma}=B_{01}+\sum_{1}^{\infty} B_{2 n, 1} \cos 2 n \gamma \\
Q_{4 \mathrm{i}}^{\gamma}=B_{04}+\sum_{1}^{\infty} B_{2 n, 4} \cos 2 n \gamma
\end{array}\right.
\end{aligned}
$$

$A^{\gamma}$ et $B^{\gamma}$ s'écrivent donc :

$$
\left\{\begin{aligned}
A^{\gamma}=a^{\prime} \frac{1}{3}+a_{1}^{\prime} A_{01} & +a_{2}^{\prime} A_{04}+\left(a_{1}^{\prime} A_{21}+a_{2}^{\prime} A_{24}\right) \cos 2 \gamma \\
B^{\gamma}=b^{\prime} \frac{1}{4}+b_{1}^{\prime} B_{01}+ & b_{2}^{\prime} B_{04}+ \\
& +\sum_{1}^{\infty}\left(b_{1}^{\prime} B_{2 n, 1}+b_{2}^{\prime} B_{2 n, 4}\right) \cos 2 n \gamma .
\end{aligned}\right.
$$

L'expérience donne les variations $A(\gamma)$ et $B(\gamma)$ des coefficients macroscopiques de la loi de Rayleigh en fonction de $\gamma$. Elle donne également $A(t)$ et $B(t)$, c'est-àdire les mêmes coefficients dans le cas de la désaimantation tournante. 
Développons également en série de Fourier $A(\gamma)$ et $B(\gamma)$. Nous obtenons :

$$
\begin{aligned}
& A(\gamma)=A_{0}+A_{2} \cos 2 \gamma+A_{4} \cos 4 \gamma+\cdots \\
& B(\gamma)=B_{0}+B_{2} \cos 2 \gamma+B_{4} \cos 4 \gamma+\cdots .
\end{aligned}
$$

En identifiant terme à terme les deux expressions précédentes, il vient :

$$
\begin{aligned}
& \left\{\begin{array}{l}
A_{0}=a^{\prime} \frac{1}{3}+a_{1}^{\prime} A_{01}+a_{2}^{\prime} A_{04}=A^{t} \\
A_{2}=a_{1}^{\prime} A_{21}+a_{2}^{\prime} A_{24} \\
A_{4}=0 \ldots \ldots \ldots \ldots
\end{array}\right. \\
& \left\{\begin{array}{l}
B_{0}=b^{\prime} \frac{1}{4}+b_{1}^{\prime} B_{02}+b_{2}^{\prime} B_{04}=B^{t} \\
B_{2}=b_{1}^{\prime} B_{21}+b_{2}^{\prime} B_{24} \\
B_{4}=b_{1}^{\prime} B_{41}+b_{2}^{\prime} B_{42} \\
\cdots \ldots \ldots \ldots
\end{array}\right.
\end{aligned}
$$

La seule connaissance de $A(\gamma), B(\gamma)$ pour une désaimantation uniaxiale et de $A(t)$ et $B(t)$ pour une désaimantation tournante ne suffit pas à déterminer toutes les constantes introduites. Elle permet seulement de déterminer $b^{\prime}, b_{1}^{\prime}$ et $b_{2}^{\prime}$ c'est-à-dire la partie irréversible de l'aimantation. Pour essayer d'aller plus loin il est nécessaire de faire une hypothèse complémentaire. Les constantes $a_{1}, b_{1}$ et $a_{2}, b_{2}$ relatives aux parois à $180^{\circ}$ et $90^{\circ}$ diffèrent d'une part à cause des grandeurs caractéristiques attachées à chaque type de paroi, d'autre part à cause du module de leur vecteur polarisation : $2 J_{\mathrm{s}}$ et $\sqrt{2} J_{\mathrm{s}}$. Nous supposerons en première approximation qu'elles ne diffèrent que par l'amplitude de leur vecteur polarisation $\left({ }^{2}\right)$ c'est-à-dire que l'on a :

$$
\begin{array}{ll}
a_{1}=2 a_{0} & a_{2}=\sqrt{2} a_{0} \\
b_{1}=2 b_{0} & b_{2}=\sqrt{2} b_{0} .
\end{array}
$$

On peut alors déterminer : $k, a^{\prime}, b^{\prime}$ et les rapports $a_{0} / b_{0}$ et $a^{\prime} / b^{\prime}$. Ces derniers sont respectivement proportionnels aux champs coercitifs de la nouvelle phase I et de la phase II (cf. L. Néel [4]).

2.3 CAS GÉNÉRAL : DÉSAIMANTATION DE FAIBLE AMPLITUDE $\mathfrak{H}_{\mathrm{m}}<3 \mathrm{H}_{\mathrm{c}}$ - Essai d'interprétation de l'évolution de l'anisotropie de désaimantation en fonction de l'amplitude maximale $H_{\mathrm{m}}$ du champ de désaimantation. - Après avoir appliqué à l'échantillon une désaimantation tournante d'amplitude $\mathscr{H}_{\mathrm{m}}>3 H_{\mathrm{c}}$, qui conduit à un état isotrope reproductible dans le plan $Z O X$, on introduit progressivement l'anisotropie de désaimantation en faisant des désaimantations uniaxiales d'amplitude maximale croissante. Après chaque désaimantation uniaxiale on retourne à l'état isotrope de référence. On étudie l'évolution de $A(\gamma)$ et $B(\gamma)$ en

(2) Remarque. - Cette hypothèse restrictive sera d'autant plus justifiée qu'un type de paroi aura un rôle prépondérant ; c'est bien ce que montre l'ensemble des résultats expérimentaux. fonction de $\mathscr{H}_{\mathrm{m}}$. C'est cette évolution que nous nous proposons d'interpréter.

Dans la phase I, il y a 6 types de parois, 3 à $180^{\circ}$ et 3 à $90^{\circ}$. Dans la phase II il intervient seulement une seule espèce de paroi moyenne. L'écriture de la loi d'aimantation macroscopique fait intervenir 7 types de parois et leurs populations respectives (cf. Tableau II). Le problème serait résolu si nous savions déterminer ces populations et leur évolution en fonction de $\mathfrak{H}_{\mathrm{m}}$. La seule connaissance des courbes de première aimantation ne permet pas de résoudre le problème ; cependant, il est possible, à partir des variations macroscopiques de $A(\gamma)$ et $B(\gamma)$ en fonction de $\mathcal{H}_{\mathrm{m}}$ d'obtenir des renseignements sur ces populations et leur évolution.

Considérons les variations relatives:

$$
\frac{Q^{\gamma}-Q_{\min }}{Q_{\max }-Q_{\min }}
$$

des $Q^{\gamma}$ ayant trait aux 6 types de parois de la phase I $\left(Q_{k \mathbf{r}}^{\gamma}\right.$ et $Q_{k \mathbf{i}}^{\gamma}$ ). On constate qu'il apparaît 3 groupes de variations qui sont relatifs aux parois 1,$4 ; 2,5$ et 3,6 , c'est-à-dire aux parois d'orientation favorable, moins favorable et défavorable par rapport à la direction du champ de désaimantation. Nous les noterons respectivement I, II et III. Les figures 7 et 8 représentent les

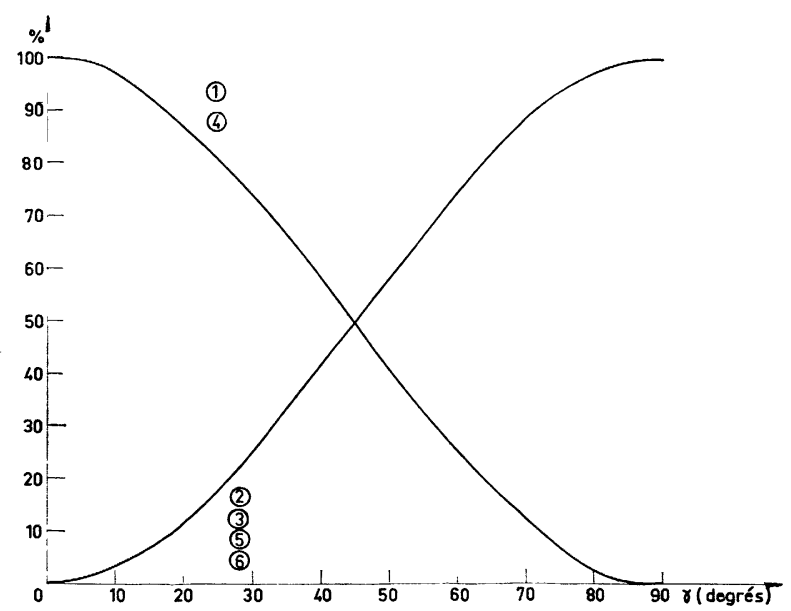

FIG. 7. - Variations relatives des $Q_{k r}^{\gamma}$ en fonction de $\gamma$.

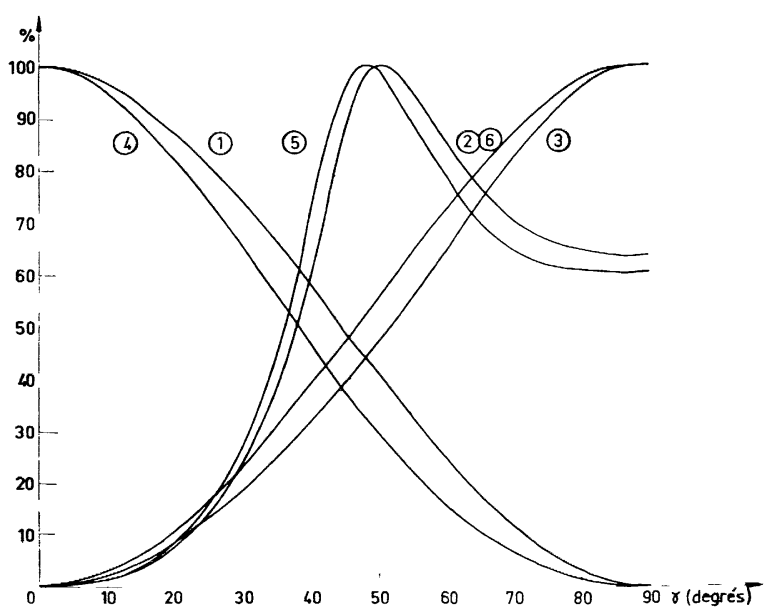

FIG. 8. - Variations relatives des $Q_{k \text { i }}^{\gamma}$ en fonction de $\gamma$. 


\section{TABleaU IV}

\begin{tabular}{llll} 
& \multicolumn{3}{c}{ I } \\
$Q_{\text {r }}$ & 1 & \multicolumn{2}{c}{4} \\
$h_{2}$ & 0,276 & 0 & 0,099 \\
$Q_{\text {i }}$ & 1 & I & 4 \\
$h_{2}$ & 0,260 & 0 & 0,100 \\
$h_{4}$ & 0,002 & & 0,013
\end{tabular}

variations relatives des divers $Q_{\mathrm{kr}}^{\gamma}$ et $Q_{\mathrm{k} \text { i }}^{\gamma}$. Le tableau IV donne le signe et les valeurs de leurs harmoniques. Dans le développement des $Q_{k \mathrm{r}}^{\gamma}$ il y a seulement le terme d'ordre zéro et l'harmonique 2 , alors que dans celui des $Q_{k \mathrm{i}}^{\gamma}$ il y a le terme constant et tous les harmoniques pairs. Pour la partie irréversible, nous nous bornerons aux harmoniques 2 et 4 qui sont les seuls que nous sachions déterminer expérimentalement avec une précision acceptable.

Remarquons que pour $\mathscr{H}_{\mathrm{m}}=0$ les populations sont les mêmes pour tous les types de parois. Ce fait a pour conséquence, à cause des propriétés des $Q$, d'entraîner pour $\mathfrak{H}_{\mathrm{m}}=0$ la nullité de tous les harmoniques sauf celui de rang zéro. Dès lors, il devient possible de prévoir l'évolution qualitative des divers harmoniques de la partie réversible et irréversible de l'aimantation, compte tenu de l'hypothèse que nous avons faite sur l'évolution des types de parois lors de l'élaboration du modèle. L'harmonique 2 de la partie réversible doit croître rapidement depuis zéro, ce qui correspond à la disparition des parois défavorables du type III $(3,6)$ qui ont une contribution négative importante, pour croître ensuite plus lentement avec $\mathcal{H}_{\mathrm{m}}$ et tendre vers une saturation correspondant à une population de parois du type $\mathbf{I}(1,4)$. L'harmonique 2 de la partie irréversible doit croître avec le champ et rester toujours positif, sa valeur limite étant celle d'une population pure du type I. L'harmonique 4 de la partie irréversible peut devenir négatif quand les parois du type III disparaissent ; l'approche à la limite doit se faire moins rapidement que celle de l'harmonique 2 à cause des contributions négatives importantes des parois du type II $(2,5)$.

La confrontation de ces considérations avec l'expérience doit nous permettre de confirmer ou d'infirmer nos hypothèses relatives aux mécanismes de désaimantation.

Comme les données expérimentales ne nous permettent pas de déterminer les 6 populations, nous sommes amenés à faire des approximations. Nous pouvons :

- soit regrouper les parois en 3 types et prendre pour chaque type une variation moyenne en fonction de $\gamma$;
II

\begin{tabular}{ccc}
\multicolumn{3}{c}{ III } \\
3 & & 6 \\
$-0,218$ & $<0$ & $-0,071$ \\
3 & III & 6 \\
$-0,202$ & $<0$ & $-0,064$ \\
0,011 & $>0$ & 0,007
\end{tabular}

- soit ne considérer qu'un seul type de parois si les résultats expérimentaux nous le suggèrent.

Or, l'expérience montre le rôle prépondérant des parois à $90^{\circ}$ tant dans les désaimantations de forte amplitude $\mathscr{H}_{\mathrm{m}}>3 H_{\mathrm{c}}$ que dans celles de faible amplitude. Nous adopterons donc ce dernier point de vue. Nous cherchons à déterminer trois proportions telles que $P_{\mathrm{I}}+P_{\mathrm{II}}+P_{\mathrm{III}}=1$ en exprimant que les harmoniques de $A(\gamma)$ et $B(\gamma)$ sont des combinaisons linéaires des lois relatives aux parois du type 4, 5, 6 . Cette détermination n'est pas possible pour la partie réversible, car nous n'avons qu'une seule relation due à l'harmonique 2 ; par contre, elle est possible pour la partie irréversible.

Pour conclure. - Nous voyons que l'étude des désaimantations de faible amplitude nous permet non seulement de tester nos hypothèses sur les mécanismes de désaimantation, mais également d'avoir une idée de l'évolution des pourcentages des divers types de parois jouant un rôle prépondérant dans l'évolution de l'anisotropie de la désaimantation en fonction de $\mathfrak{H}_{\mathrm{m}}$.

2.4 INFLuence DES MÉCANISMES DE ROTATION. Dans ce qui précède, nous avons supposé que les vecteurs $\mathbf{J}_{\mathrm{s}}$ restaient suivant une direction de facile aimantation et ne tournaient pas sous l'influence du champ appliqué. Une légère rotation de ces vecteurs autour de leur position d'équilibre a deux conséquences : il apparaît d'une part une variation réversible d'aimantation proportionnelle au champ magnétique appliqué et suivant celui-ci, d'autre part la répartition des vecteurs $\mathbf{J}_{\mathrm{s}}$ change, ce qui entraîne une modification de la répartition des vecteurs polarisation qui se répercute sur les variations d'aimantation dues aux déplacements de parois. Nous analysons successivement ces deux conséquences dans l'annexe 1 .

On peut montrer que l'influence des rotations sur la répartition des vecteurs polarisation, qui n'affecte que les variations d'aimantation consécutives aux déplacements de parois à $90^{\circ}$, est un effet négligeable. Il suffit pour s'en convaincre de remarquer que les nouvelles quantités $Q$ introduites seront du même ordre de 
grandeur que les précédentes et que leur influence sera toujours diminuée par le facteur

$$
\frac{J_{\mathrm{s}}^{2}}{2 \sqrt{2} K_{1}}<H>\left({ }^{3}\right) \text {. }
$$

En particulier, l'harmonique 4 qui apparaît dans les termes correctifs de $Q_{k \mathrm{r}}^{\gamma}$ c'est-à-dire dans $A(\gamma)$ est très petit. Il nous reste à voir quelle sera l'influence de la susceptibilité rotationnelle initiale. Celle-ci fait apparaître dans $A(\gamma)$ tous les harmoniques pairs. La partie constante de $A(\gamma)$ est modifiée. Le terme isotrope affecte la phase II, le terme important étant :

$$
\frac{J_{\mathrm{s}}^{2}}{3 K_{1}}<H>\text {. }
$$

Le terme anisotrope $\left(\chi_{\text {rot }}\right)_{i}$ affecte la phase $I$, dans celuici les termes en

$$
\frac{J_{\mathrm{s}}^{2}}{2 K_{1}}
$$

sont prépondérants. L'harmonique 2 de $A(\gamma)$ sera essentiellement modifiée par les termes en

$$
\frac{J_{\mathrm{s}}^{2}}{2 K_{1}}
$$

de $\left(\chi_{\text {rot }}\right)_{i}$. L'harmonique 4 de $A(\gamma)$ provient du dernier terme de $\left(\chi_{\text {rot }}\right)_{i}$. Il sera toujours très petit à cause du facteur

$$
\frac{J_{\mathrm{s}}^{3}}{4 K_{1}^{2}}<H>
$$

Physiquement, il provient de l'évolution de la déformation de la répartition des vecteurs $\mathbf{J}_{\mathrm{s}}$ par le champ magnétisant. Les vecteurs $\mathbf{J}_{\mathrm{s}}$ du type 2 (moyennement proches) donnent une contribution négative à cet harmonique.

Toutefois, il ne faut pas perdre de vue que ces conclusions ne sont valables que pour un matériau du type fer ayant une forte constante d'anisotropie. On arriverait à des conclusions très différentes avec le nickel. Nous montrerons ultérieurement qu'avec l'échantillon étudié les mécanismes de rotation n'affectent pratiquement qu'assez peu la partie constante de $A$ et ne modifient pas de façon sensible les variations angulaires.

3. Vérification expérimentale $\left({ }^{4}\right)$. - Nous préciserons d'abord la méthode de mesure et les précautions que nous avons dî prendre pour mener à bien cette étude. Nous interpréterons ensuite nos résultats expérimentaux

(3) La déformation de la répartition des polarisations varie avec $\boldsymbol{H}$ quand on aimante la substance et il faut considérer une action intégrée dans la gamme de variation du champ de mesure. Nous désignerons par $\langle H\rangle$ cette fonction de $H$.

(4) Remarque. - Les mesures que nous avons utilisées dans ce chapitre sont celles de la thèse de Z. Blazek [33]. dans le cadre du modèle. - Nous traiterons d'abord des désaimantations de forte amplitude $\left(\mathscr{H}_{\mathrm{m}}>3 H_{\mathrm{c}}\right)$ puis nous reviendrons aux désaimantations de faible amplitude $\left(\mathscr{H}_{\mathrm{m}}<3 H_{\mathrm{c}}\right)$ et nous verrons dans quelle mesure elles confirment les hypothèses que nous avions faites au sujet des mécanismes de désaimantation.

3.1 Méthode de Mesure. - Pour faire les mesures d'anisotropie de la désaimantation nous avons utilisé un échantillon ayant la forme d'un disque mince : $\varnothing=60 \mathrm{~mm}, e=1,2 \mathrm{~mm}$. Nous l'avons assimilé à un ellipsoïde de révolution aplati dont le plan équatorial $Z O X$ est confondu avec le plan du disque. Nous avons adopté comme coefficient de champ démagnétisant celui de l'ellipsoïde, soit $N=0,20$ dans le plan du disque. Un ensemble de bobines permet d'appliquer le champ magnétisant $\mathbf{H}$ et le champ alternatif de désaimantation $\mathfrak{H}$ dans une direction quelconque du plan $Z O X$. Nous avons mesuré l'aimantation à l'aide d'un fluxmètre électronique [27, 28] complété par un dispositif permettant une compensation partielle ou totale des variations de flux dues à l'aimantation réversible. Dans nos expériences, l'aimantation réversible représentait $97,5 \%$ de l'aimantation totale. Dès lors, nous pouvions suivre l'évolution de l'aimantation de l'échantillon et mesurer avec précision de très faibles variations d'aimantation irréversible.

Nous avons obtenu un état de référence isotrope dans le plan du disque en utilisant une désaimantation tournante dont l'amplitude maximale $\mathcal{H}_{\mathrm{m}}$ était quelques fois $H_{\mathrm{c}}$. Cet état était parfaitement reproductible. Nous avons ensuite introduit l'anisotropie de désaimantation en faisant une désaimantation alternative de direction et d'amplitude maximale déterminées. Nous appliquons alors le champ magnétisant dans la direction choisie et nous relevons la courbe de première aimantation suivant cette même direction.

Comme l'aimantation dépend du temps à cause $\mathrm{du}$ traînage de fluctuations thermiques, et d'un éventuel traînage de diffusion, il est nécessaire de minuter soigneusement les expériences. Après avoir fait les deux désaimantations, nous appliquons à l'échantillon une montée linéaire en champ et nous mesurons l'aimantation résultante. Nous avons répété les expériences en fonction de l'amplitude maximale $\mathscr{H}_{\mathrm{m}} \mathrm{du}$ champ de désaimantation et de l'angle $\gamma$ que font le champ magnétisant et le champ de désaimantation.

Nous n'avons pas été gênés dans nos mesures par le traînage de diffusion ; il n'en est pas de même du traînage de fluctuations thermiques [29]. Nous en avons d'ailleurs fait une étude systématique sur notre échantillon. Pour un processus de montée linéaire en champ nous ne savons pas calculer rigoureusement l'influence du traînage de fluctuations thermiques, les relations habituelles [30] n'étant plus applicables. Nous pensons cependant que pour l'usage que nous avons fait de ces mesures il était plus important de rendre ce traînage reproductible que de chiffrer rigoureusement son influence. Dans le cas d'un relevé point par point des 
courbes d'aimantation il devient possible d'en tenir compte rigoureusement [30], [26].

Pour vérifier le modèle, le choix de la substance n'est pas critique. Nous avons utilisé un polycristal de structure cubique sans orientation préférentielle notable et de comportement magnétique analogue à celui du fer. $\mathrm{Sa}$ structure en domaines doit être suffisamment complexe pour qu'on puisse lui appliquer valablement le traitement statistique qui est à la base des lois de Rayleigh. Nous avons utilisé un acier de composition en poids :

$\mathrm{Fe} 93,4 \%$; Ni 4,3\%; Cr $1,5 \%$; Mo $0,5 \%$; C $0.3 \%$.

Nous l'avons recuit en atmosphère d'hydrogène pendant $1 \mathrm{~h}$ à $650^{\circ} \mathrm{C}$ et refroidi lentement en $24 \mathrm{~h}$. Ce traitement thermique conduit à un champ coercitif de 14,7 Oe dans le plan $Z O X$.

Nous avons vérifié sur cet échantillon la validité des lois de Rayleigh. Dans un domaine de champ compris entre $70 \times 10^{-3}$ et 2,2 Oe les écarts à la droite représentant la susceptibilité en fonction du champ restent inférieurs à $10^{-3}$. Au-dessous de $70 \mathrm{mOe}$ la susceptibilité décroît plus vite que ne le prévoit la loi de Rayleigh. Ce phénomène a été constaté par d'autres auteurs [2, 3]. Il peut être attribué, entre autres choses, au traînage de diffusion et au traînage de fluctuations thermiques. Au-delà de 2,2 Oe on observe le phénomène habituel : la susceptibilité croît plus vite que ne l'indique la loi. Nous avons déterminé les constantes $A$ et $B$ relatives à cet intervalle de champ en ajustant la susceptibilité $J / H$ sur une droite par une méthode de moindres carrés. La reproductibilité de nos mesures était supérieure à $\pm 10^{-3}$ pour $A$ et $\pm 2,5 \times 10^{-2}$ pour $B$. Quant aux valeurs de $A$ et $B$ relevées après une désaimantation isotrope, on avait :

$$
A=4,405 \text { uém } \cdot \mathrm{Oe}^{-1}, \quad B=0,112 \text { uém. } \mathrm{Oe}^{-2} \text {. }
$$

En ce qui concerne les valeurs absolues, la précision est limitée par les déterminations du coefficient de champ démagnétisant et de la surface active des bobines.

3.2 VÉRIfiCATION DU MODÈle. - 3.2.1 Désaimantation de grande amplitude $\mathcal{H}_{\mathrm{m}}>3 H_{\mathrm{c}}$ - - 1) Pour confronter la théorie avec l'expérience, nous comparerons d'abord les courbes $f(\gamma)$ et $g(\gamma)$ donnant les variations relatives de $A(\gamma)$ et $B(\gamma)$ avec les courbes théoriques correspondantes :

$$
f(\gamma)=\frac{A^{\gamma}-A^{\perp}}{A^{\|}-A^{\perp}}, \quad g(\gamma)=\frac{B^{\gamma}-B^{\perp}}{B^{\|}-B^{\perp}}\left({ }^{5}\right) .
$$

En ce qui concerne la partie réversible de l'aimantation, les parois à $180^{\circ}$ et les parois à $90^{\circ}$ donnent les mêmes variations relatives. Il n'en est plus de même en ce qui concerne la partie irréversible. La figure 9 relative à la

(5) Remarque. $-A^{\|}=A^{\gamma}$ pour $\gamma=0 ; A^{\perp}=A^{\gamma}$ pour $\gamma=\pi / 2$. partie réversible montre un écart systématique faible $\left(7 \%\right.$ pour $\left.\gamma=45^{\circ}\right)$ entre la théorie et l'expérience. Nous reviendrons ultérieurement sur ce point. La figure 10 relative à la partie irréversible montre que la courbe expérimentale se rapproche beaucoup plus de la courbe théorique relative aux parois à $90^{\circ}$ que de celle des parois à $180^{\circ}$. Ce fait nous laisse penser que le rôle des parois à $90^{\circ}$ est prépondérant dans l'anisotropie de désaimantation.

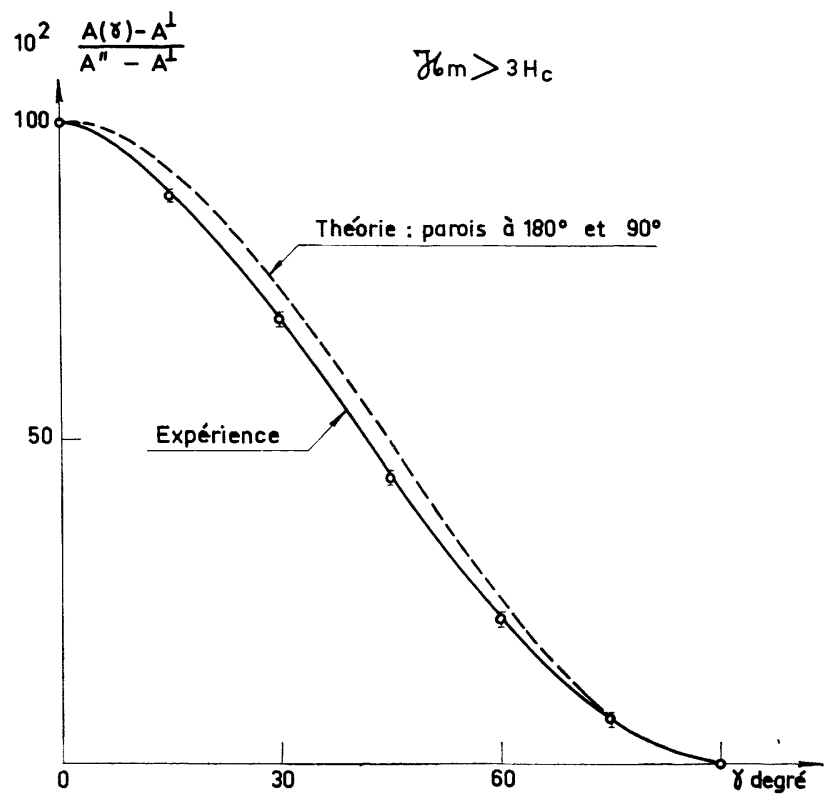

FIG. 9. - Variations relatives de $A(\gamma)$ en fonction de $\gamma$. Comparaison de la théorie avec l'expérience pour les parois à $180^{\circ}$, et à $90^{\circ}$. Cas où $\mathfrak{K}_{\mathrm{m}}>3 H_{\mathrm{c}}$.

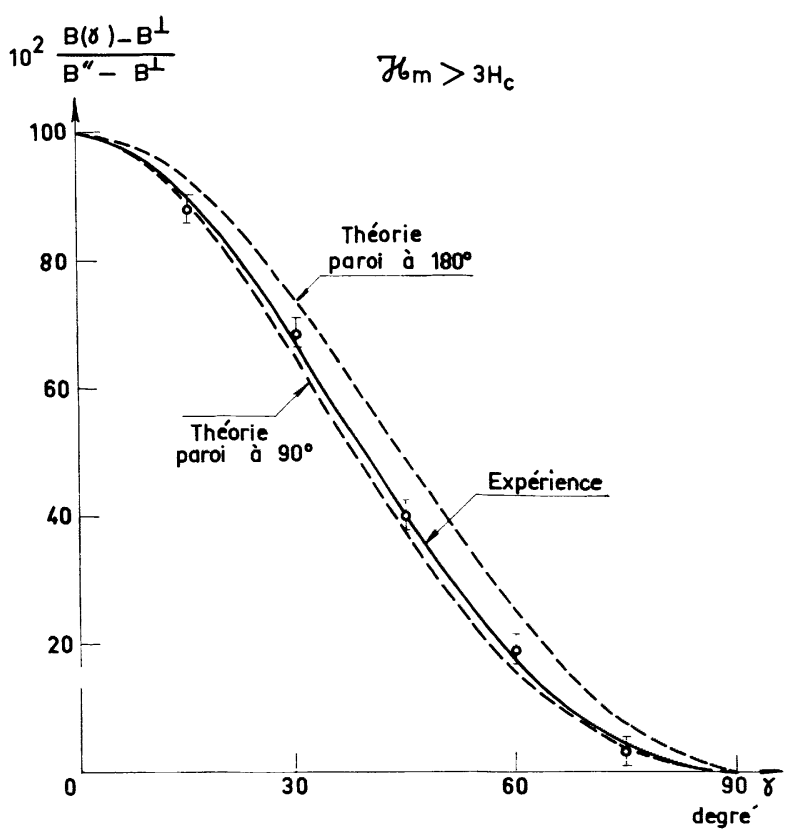

FIG. 10. - Variations relatives de $B(\gamma)$ en fonction de $\gamma$. Comparaison de la théorie avec l'expérience pour les parois à $180^{\circ}$ et à $90^{\circ}$. Cas où $\mathcal{H}_{\mathrm{m}}>3 H_{\mathrm{c}}$. 
2) Résultats relatifs aux développements en série de Fourier. Nous avons utilisé les résultats relatifs à l'intervalle $0-\pi / 2$ complété par symétrie. Nous disposons de deux séries de mesures de $A(\gamma)$ et $B(\gamma)$ en fonction de l'amplitude du champ de désaimantation. Pour la première nous n'avons pas dépassé les champs internes de 50 Oe alors que nous avons atteint plus de 100 Oe pour la seconde.

\section{TABLEAU $\mathrm{V}$}

\begin{tabular}{|c|c|c|c|c|c|c|}
\hline $\begin{array}{l}\mathfrak{H e}_{\mathrm{m}} \\
\mathrm{Oe}\end{array}$ & $A_{0}$ & $A_{2}$ & $A_{4}$ & $B_{0}$ & $B_{2}$ & $B_{4}$ \\
\hline 47 & 418 & 0,248 & 0,01 & $0,117_{5}(1)$ & 0,026 & \\
\hline & & 0,252 & 0,020 & $0,111_{5}$ & 0,027 & \\
\hline 100 & 4,430 & 0,258 & 0,021 & $0,111_{8}$ & 0,029 & 0,005 \\
\hline
\end{tabular}

Nos résultats sont résumés dans le tableau V. (1)et (2) proviennent de deux séries de mesures ayant été faites à plusieurs mois d'intervalle; elles permettent de juger de la reproductibilité. Il est difficile de comparer (2) et (3), car même au-delà de $3 H_{\mathrm{c}}$ le phénomène évolue encore lentement en fonction de $\mathscr{H}_{\mathrm{m}}$. Cette remarque est valable pour l'ensemble des déterminations relatives aux désaimantations de grande amplitude. L'examen du tableau $\mathrm{V}$ montre que la reproductibilité sur $A_{0}$ et $A_{2}$ est bonne : $\sim 1,6 \times 10^{-3}$. Elle est un peu moins bonne pour $B_{0}$ et $B_{2}$, ce qui est normal, car on mesure $B$ avec une moins bonne précision que $A$. La reproductibilité sur le terme $B_{4}$ est nettement moins bonne: $\sim 16 \times 10^{-2}$. Ceci est dû au fait que ce terme est déjà très sensible aux imprécisions de mesure. Au-delà de l'harmonique 4 les termes ne sont plus significatifs.

\section{TABLEAU VI}

\begin{tabular}{|c|c|c|c|c|}
\hline $\begin{array}{c}\mathcal{H}_{\mathrm{m}} \\
\mathrm{Oe}\end{array}$ & $A_{0}$ & $A^{t}$ & $B_{0}$ & $B^{t}$ \\
\hline (1) 47 & 4,418 & 4,417 & $0,117_{5}\left({ }^{1}\right)$ & $0,112_{1}$ \\
\hline (2) 50 & 4,425 & 4,418 & $0,111_{5}$ & $0,112_{2}$ \\
\hline (3) 100 & 4,430 & 4,425 & $0,111_{8}$ & $0,112_{4}$ \\
\hline
\end{tabular}

La théorie prévoit $A_{0}=A_{t} ; B_{0}=B_{t}$. Le tableau VI montre que cette conséquence est particulièrement bien vérifiée par l'expérience.

3) Détermination des constantes $b^{\prime}, b_{1}^{\prime}, b_{2}^{\prime}$ ayant trait respectivement à une paroi moyenne, à une paroi à $180^{\circ}$ et à une paroi à $90^{\circ}$.

Il faut s'attendre ici à une certaine dispersion des résultats relatifs à $b_{1}^{\prime}$ et $b_{2}^{\prime}$ due au fait que l'incertitude sur les harmoniques de $B$ se répercute de façon notable sur ces valeurs à cause de la forme des équations. Le tableau VII donne les résultats obtenus.

4) L'approximation $a_{1}=2 a_{0}, \quad b_{1}=2 b_{0}$; $a_{2}=\sqrt{2} a_{0}, b_{2}=\sqrt{2} b_{0}$ nous permet de calculer $k, a^{\prime}$

(1) Remarque. - Compte tenu d'autres mesures, il semble que la valeur de $B_{0}$ relative à 47 Oe soit aberrante. et les rapports $b_{0} / a_{0}$ et $b^{\prime} / a^{\prime}$ relatifs aux deux phases du cristal.

\section{TABLEAU VII}

\begin{tabular}{|c|c|c|c|}
\hline $\begin{array}{l}\mathcal{H}_{\mathrm{m}} \\
\mathrm{Oe}\end{array}$ & $b_{1}^{\prime}$ & $b_{2}^{\prime}$ & $b^{\prime}$ \\
\hline (1) 47 & 0,038 & 0,130 & 0,176 \\
\hline (2) 50 & 0,027 & 0,133 & 0,174 \\
\hline (3) 100 & 0,070 & 0,472 & 0,184 \\
\hline
\end{tabular}

TABleaU VIII

\begin{tabular}{|c|c|c|c|c|c|c|}
\hline $\begin{array}{l}\mathscr{H E}_{\mathbf{m}} \\
\mathrm{Oe}\end{array}$ & $k$ & $a_{0} \varepsilon$ & $b_{0} \varepsilon$ & $a^{\prime}$ & $a_{0} / b_{0}$ & $a^{\prime} / b^{\prime}$ \\
\hline 1) 47 & $\overline{0,86}$ & $\overline{1,49}$ & 0,238 & $11,2_{6}$ & $6,2_{8}$ & 6 \\
\hline (2) 50 & 0,87 & 1,12 & 0,106 & 11,8 & $10,6^{\circ}$ & 8 \\
\hline (3) 100 & 0,90 & 1,43 & 0,369 & $11,2_{5}$ & 3,89 & \\
\hline
\end{tabular}

Le tableau VIII donne les résultats obtenus. Nous trouvons toujours $k$ voisin de 1 , ce qui montre que les parois à $90^{\circ}$ semblent jouer un rôle prépondérant dans l'anisotropie de la désaimantation. Ce fait légitime l'approximation précédente et explique la position de la courbe expérimentale par rapport aux courbes théoriques sur la figure 10. La dispersion observée sur les valeurs du rapport $a_{0} / b_{0}$ est due aux mêmes raisons que celles que nous avons invoquées au $\S 3.2 .2$. Dans son mémoire sur les lois de Rayleigh, L. Néel [4] montre que le champ coercitif d'une substance polydomaine ne contenant qu'un seul type de parois moyennes caractérisées par deux constantes élémentaires $a$ et $b$ est proportionnel au rapport $a / b$. Il en résulte que les champs coercitifs de la nouvelle phase I et de la phase II sont respectivement proportionnels à $a_{0} / b_{0}$ et à $a^{\prime} / b^{\prime}$; en effet :

$$
\frac{a^{\prime}}{b^{\prime}}=\frac{a(1-\varepsilon)}{b(1-\varepsilon)}=\frac{a}{b}
$$

La phase II est plus dure que la phase I, le rapport des champs coercitifs allant de 6 à 18 . Nous faisons cependant les réserves d'usage sur ce dernier chiffre ; seules des moyennes sur un grand nombre d'expériences permettraient de conclure avec certitude.

5) Influence des rotations. L'acier que nous avons étudié contient en poids : $4,3 \%$ de $\mathrm{Ni}, 1,5 \%$ de $\mathrm{Cr}$, $0,5 \%$ de Mo et $0,3 \%$ de $\mathrm{C}$, ce dernier n'étant vraisemblablement pas en solution. En admettant que le Ni, le $\mathrm{Cr}$ et le Mo soient en solution et que leurs contributions à l'abaissement de $K_{1}$ s'ajoutent, on obtient d'après [31] et [32] $K_{1} \sim 3,5 \times 10^{5} \mathrm{erg} . \mathrm{cm}^{-3}$, alors que ce même $K_{1}$ est de l'ordre de $4,6 \times 10^{5} \mathrm{erg} . \mathrm{cm}^{-3}$ pour le fer pur. Il s'agit là bien sûr d'une estimation très grossière, mais suffisante pour déterminer l'ordre de grandeur des termes. En prenant pour $J_{\mathrm{s}}$ la valeur $\mathrm{du}$ fer pur, on obtient les ordres de grandeur du tableau IX.

Une partie du terme constant de $A(\gamma)$ aura les rota- 
tions pour origine. Le terme le plus important provient de $\left(\chi_{\text {rot }}\right)_{\text {isotrope }}$ et vaut :

$$
\frac{J_{\mathrm{s}}^{2}}{3 K_{1}} \quad \text { soit } \sim 2,7 \text { uém. } \mathrm{Oe}^{-1} \text {. }
$$

Il affecte la phase II et il faut le comparer à $a$. Nous ne connaissons pas $a$, mais nous avons une limite inférieure puisque $a^{\prime}=(1-\varepsilon) a$ est connu. Or, comme $a^{\prime} \sim 11,4$ uém. $\mathrm{Oe}^{-1}$, l'influence des rotations sera au maximum de $\sim 20 \%$ sur ce terme. En ce qui concerne la phase I on a en facteur le terme :

$$
\frac{J_{\mathrm{s}}^{2}}{2 K_{1}} \sim 4,2 \text { uém. } \mathrm{Oe}^{-1}
$$

il faut le comparer à $a_{1}$ et $a_{2}$ ou à $a_{0}$, valeurs que nous ne connaissons pas. Mais nous avons montré que la nouvelle phase I était beaucoup plus douce que la phase II, son champ coercitif étant entre 6 et 18 fois plus petit. Nous pouvons prendre pour ordre de grandeur de $a_{1}, a_{2}$ et $a_{0}$ la susceptibilité initiale du fer doux, soit $\sim 30$ uém. $\mathrm{Oe}^{-1}$. Comme la partie constante des termes $Q$ est de l'ordre de 0,4 il convient de comparer :

$$
0,4 \frac{J_{\mathrm{s}}^{2}}{2 K_{1}} \text { à } 30
$$

pour avoir l'influence des rotations sur ce terme. La situation est ici beaucoup plus favorable.

En ce qui concerne l'harmonique 2 de $A(\gamma)$ seule la phase I est affectée et le terme prépondérant est celui en :

$$
\frac{J_{\mathrm{s}}^{2}}{2 K_{1}}
$$

Nous sommes dans la situation précédente mais avec des valeurs des harmoniques 2 des $Q$ qui sont de l'ordre de 0,1 à 0,2 . Les autres termes sont négligeables devant le précédent car ils sont affectés du facteur :

$$
\frac{J_{\mathrm{s}}^{3}}{4 K_{1}^{2}}<H>\text { qui vaut } \sim 10^{-2} \text { uém. } \mathrm{Oe}^{-1} \text {. }
$$

La contribution aux harmoniques supérieurs est parfaitement négligeable.

\section{Tableau IX}

$\begin{array}{cccc}\frac{J_{\mathrm{s}}^{2}}{3 K_{1}} & \frac{J_{\mathrm{s}}^{2}}{2 K_{1}} & \frac{\left(J_{\mathrm{s}}\right)^{3}<H>(1)}{4 K_{1}^{2}} & \frac{J_{\mathrm{s}}}{2 \sqrt{2} K_{1}} \\ \frac{-}{2,7} & \overline{-} & \overline{0} & -01 \\ \text { uém. } \mathrm{Oe}^{-1} & \text { uém. } \mathrm{Oe}^{-1} & \text { uém. } \mathrm{Oe}^{-1} & 1,7 \times 10^{-3} \\ \text { uém. } \mathrm{Oe}^{-1}\end{array}$

Pour conclure, nous remarquerons que dans le domaine de champ utilisé la prise en considération des rotations ne change pas de façon notable nos résultats; seule la partie constante de $A(\gamma)$ est légèrement affectée,

(1) Remarque. - Nous prendrons pour $\langle H\rangle$ la valeur $H_{\mathrm{m}} / 2$ soit environ $1 \mathrm{Oe}$. mais les variations angulaires ne sont pratiquement pas modifiées. Ces considérations restent encore valables même pour des valeurs nettement plus faibles de $K_{1}$.

6) Anisotropie résiduelle de l'échantillon dans le plan $Z O X$. Dans ce qui précède, nous avons supposé un état de référence isotrope dans le plan $Z O X$. Au début de cette étude, nous avons essayé de mettre en évidence une éventuelle anisotropie dans ce plan. Pour cela nous avons fait trois séries de mesures de $A(\gamma)$ et $B(\gamma)$ :

I. L'échantillon étant dans une position déterminée (que nous avons conservée dans toute la suite de l'étude) ;

II. l'échantillon étant tourné de $\pi / 2$;

III. l'échantillon étant à nouveau dans sa position initiale.

Ces mesures nous ont permis de mettre en évidence une légère anisotropie résiduelle. Celle-ci se manifeste de façon claire sur le terme $A(\gamma)$ : l'écart relatif maximum étant de l'ordre de $6 \times 10^{-3}$ alors que l'écart relatif moyen est de $4 \times 10^{-3}$, ceci entre la position normale et la position tournée de $\pi / 2$. Ces écarts sont toujours de même signe et dépassent nettement l'irréproductibilité des mesures. Pour $B(\gamma)$ au contraire, ils sont de signes alternés et du même ordre de grandeur que l'irréproductibilité des mesures. Comme cette anisotropie affecte seulement les termes réversibles de quelques $10^{-3}$, nous n'avons pas fait d'étude systématique. Une telle étude est cependant possible.

7) Il reste à examiner le léger désaccord qui subsiste entre l'expérience et le modèle, à savoir l'existence dans le développement en série de Fourier de $A(\gamma)$ d'harmoniques pairs de rang supérieur à 2, en particulier du terme $A_{4}$ (cf. Tableau V). Ce sont ces termes qui sont responsables de l'écart systématique observé sur la figure 9. Précisons leur importance : ils représentent 3 à $5 \times 10^{-3}$ de l'aimantation réversible.

Les mécanismes de rotation donnent des termes de ce type, mais leur ordre de grandeur n'est pas convenable : ils sont environ 100 fois trop petits. Il ne faut pas chercher là leur origine. Par contre l'anisotropie résiduelle de l'échantillon peut en être responsable.

3.2.2 Cas général : désaimantation de faible amplitude $H_{\mathrm{m}}<3 H_{\mathrm{c}}$. - Rappelons brièvement les prévisions du modèle relatives à l'évolution des divers harmoniques de la partie réversible et de la partie irréversible de l'aimantation.

L'harmonique 2 de la partie réversible doit croître rapidement depuis zéro, ce qui correspond à la disparition des parois défavorables du typa III qui ont une contribution négative importante, pour croître ensuite plus lentement avec $\mathscr{H}_{\mathrm{m}}$ et tendre vers une situation correspondant à une population de parois de type I (cf. Fig. 11). L'harmonique 2 de la partie irréversible doit croître avec le champ et rester toujours positif, sa valeur limite étant celle d'une population pure de 
type I (cf. Fig. 12). L'harmonique 4 de la partie irréversible peut devenir négatif quand les parois du type III disparaissent. L'approche à la limite doit se faire moins rapidement que celle de l'harmonique 2 à cause des contributions négatives importantes des

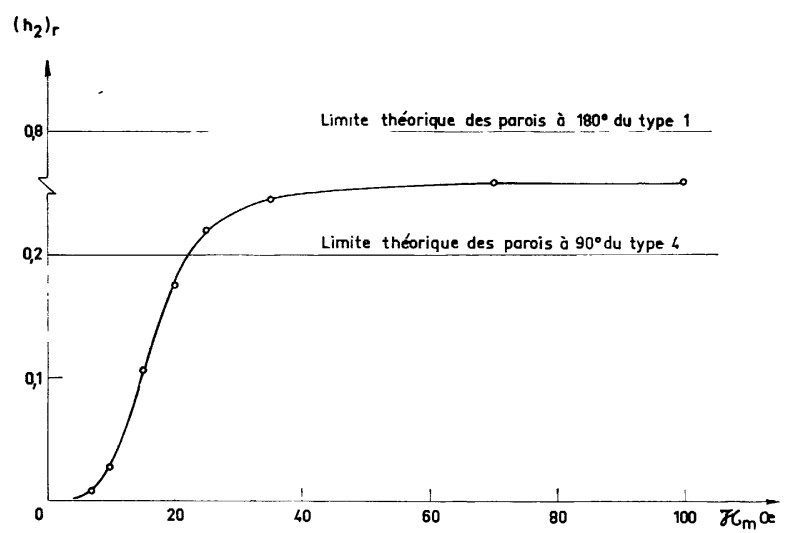

Fig. 11. - Evolution en fonction de $\mathfrak{H}_{\mathrm{m}}$ de l'harmonique 2 de la partie réversible de l'aimantation, comparée aux valeurs théoriques relatives aux parois du type 1 et 4 . Cas où $\mathcal{H}_{\mathrm{m}}<3 H_{\mathrm{c}}$.

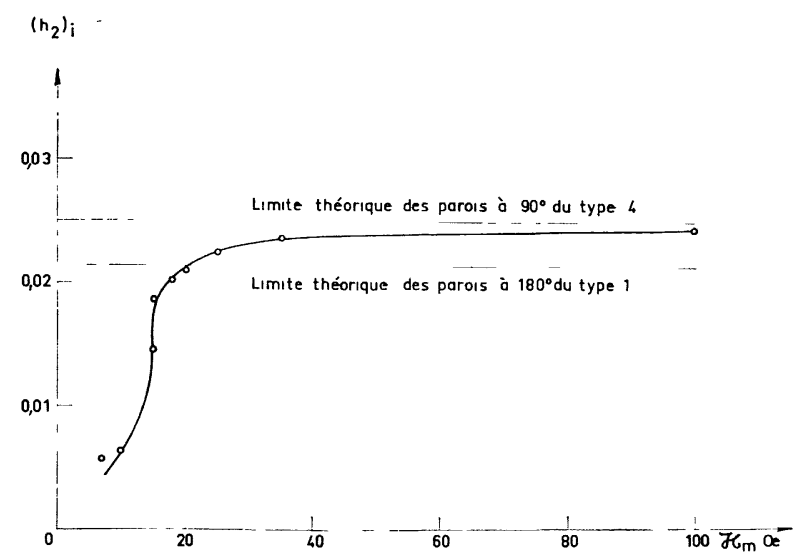

FIG. 12. - Evolution de fonction de $\mathcal{H}_{\mathrm{m}}$ de l'harmonique 2 de la partie irréversible de l'aimantation, comparée aux valeurs théoriques relatives aux parois du type 1 et 4 . Cas où $\mathscr{H}_{\mathrm{m}}<3 H_{\mathrm{c}}$.

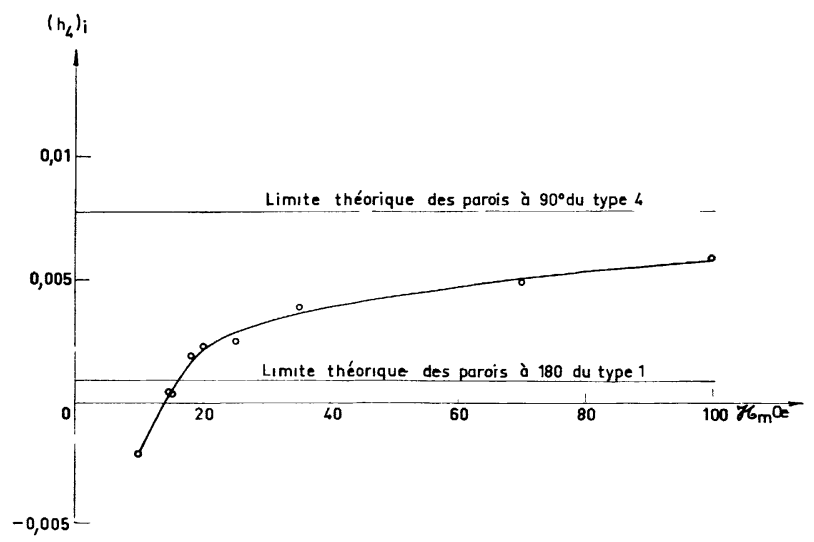

Fig. 13. - Evolution en fonction de $\mathfrak{H}$ de l'harmonique 4 de la partie irréversible de l'aimantation, comparée aux valeurs théoriques relatives aux parois du type 1 et 4 . Cas où $\mathfrak{H}_{\mathrm{m}}<3 H_{\mathrm{c}}$. parois du type II (cf. Fig. 13). Les résultats expérimentaux (Fig. 11, 12, 13) montrent que les conclusions précédentes sont particulièrement bien vérifiées, ceci est en faveur des hypothèses que nous avons faites à propos des mécanismes de désaimantation. Ces résultats confirment le rôle prépondérant des parois à $90^{\circ}$ que nous avions déjà mis en évidence à propos des désaimantations de forte amplitude : $H_{\mathrm{m}}>3 H_{\mathrm{c}}$.

Dans le cas particulier où l'on suppose que seules les parois à $90^{\circ}$ interviennent, la figure 14 donne l'évolution des populations des divers types de parois en fonction de $\mathfrak{H}_{\mathrm{m}}$, pour la partie irréversible de l'aimantation.

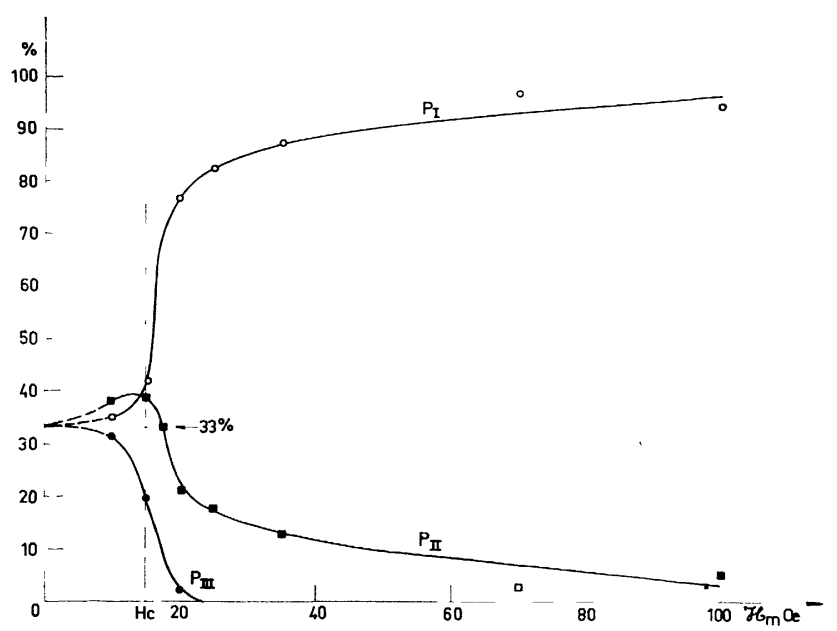

Fig. 14. - Evolution en fonction de $\mathscr{H}_{\mathrm{m}}$ des pourcentages

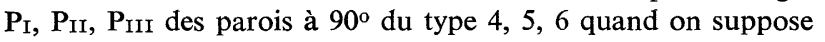
qu'elles interviennent seules dans la phase I.

4. Conclusion. - Nous avons pu dans cette étude, grâce à des mesures d'aimantation suffisamment fines et élaborées, réunir un ensemble de faits qui nous a permis de développer un modèle interprétant l'anisotropie de la désaimantation consécutive à l'application d'un champ alternatif évanescent. Ce phénomène est lié à la possibilité, au niveau du cristallite, d'un choix pour les vecteurs $\mathbf{J}_{\mathrm{s}}$ entre plusieurs axes de facile aimantation qui ne sont plus énergétiquement équivalents au cours de la désaimantation. On le rencontrera donc dans les substances cubiques, mais pas dans les substances uniaxes. Le modèle que nous avons développé suppose que la structure en domaines du matériau est suffisamment complexe pour qu'on puisse lui attribuer valablement des propriétés statistiques simples. L'expérience confirme bien les processus de désaimantation que nous avons proposés. Nous retrouvons d'ailleurs les résultats empiriques bien connus relatifs aux désaimantations alternatives et nous les justifions. Nous montrons également le rôle prépondérant que jouent les parois à $90^{\circ}$ dans l'anisotropie de la désaimantation. Or ces parois se trouvent en plus grand nombre dans les régions perturbées du cristal De plus, pour que la substance ne garde aucune mémoire d'un état désaimanté anisotrope donnée, il 
faut lui appliquer un champ suffisant pour effacer complètement la structure en domaine initiale, c'est-àdire un champ au moins égal à $H_{\mathrm{c}}$. Ces raisons nous incitent à attribuer l'origine de l'anisotropie de la désaimantation à une modification des centres de nucléation qui prennent naissance dans les régions perturbées du cristal au cours de la désaimantation. Ce sont elles qui conditionnent la structure en domaines qui dépend du type de désaimantation.

Remerciements. - Les auteurs remercient MM. J. C. Barbier et P. Brissonneau tant pour les discussions fructueuses qu'ils ont eues avec eux que pour l'intérêt avec lequel ils ont suivi ce travail.

\section{Bibliographie}

[1] Lord Rayleigh, Phil. Mag. 23 (1887) 225.

[2] Koller, A., Pfrenger, E. et Stierstat, K., J. Appl. Phys. 39 (1968) 869.

[3] Kneller, E., Ferromagnetismus (1962) 593.

[4] NéEL, L., Cah. Phys. 12 (1942) 1. NéEL, L., Cah. Phys. 13 (1943) 18.

[5] Bonnet, G., J. Physique 11 (1950) 49.

[6] Fahlenbrach, H., Sixtus, K., Z. Metal. 40 (1949) 187.

[7] Fahlenbrach, H., Arch. Eisenhüttenw. 23 (1952) 47.

[8] SOMmerkorn, G., Krupp Tech. Mitt. 13 (1955) 71.

[9] Broz, J., Stenberg, J., Czech. J. Phys. 5 (1955) 425.

[10] Smolenski, A., Br. J. Appl. Phys. 12 (1961) 81.

[11] Néel, L., Phil. Mag. Suppl. 4 (1955) 191.

[12] Chikazumi, S., Physics of Magnetism (1964) 258.

[13] SNOEK, J. L., FAST, J. F., Nature 161 (1948) 887.

[14] KNeller, E., Ferromagnetismus (1962) 593.

[15] Klitzing, K. H. et Zentgraf, G., Z. Phys. 180 (1964) 478.

[16] NéEL, L., Notes inédites exposées au C. N. R. S., Grenoble (1959).

[17] Barbier, J. C., Ferlin Guion, B., J. Appl. Phys. Suppl. 33 (1962) 1226.
[18] Barbier, J. C., Ruby, H. et Vergne, R., C. R. Hebd. Séan. Acad. Sci. 260 (1964) 3014.

[19] Ruby, H., thèse de $3^{\mathrm{e}}$ cycle, Grenoble (1965).

[20] Guion, B., D. E. S. Grenoble (1961).

[21] Covo, J., Thèse de $3^{\mathrm{e}}$ cycle, Grenoble (1960).

[22] J. Covo, Notes inédites.

[23] Abel's, V. R., Izv. Akad. Nauk., SSSR, Ser. fiz. 25 (1961) 1452.

[24] Angel, Y., Acta Electron. 7 (1963) 7 et 119.

[25] Bonnefous, J., Acta Electron. 11 (1968) 7.

[26] Vergne, R., Porteseil, J. L. et Blazek, Z., Phys. Stat. Sol. (a) 25 (1974) 171-184.

[27] Porteseil, J. L., Thèse $3^{\mathrm{e}}$ cycle, Grenoble (1969).

[28] Vergne, R., Porteseil, J. L., Revue Phys. Appl. 6 (1971) 95.

[29] Barbier, J. C., thèse d'Etat, Grenoble (1953).

[30] NéEL, L., J. Physique 11 (1950) 49. NÉEL, L., J. Physique 12 (1951) 339.

[31] KNeller, E., Ferromagnetismus (1962).

[32] Hall, R. C., J. Appl. Phys. 31 (1960) 1037.

[33] BlazeK, Z., Thèse Docteur ingénieur, Grenoble 1972.

\section{ANNEXE I}

Influence des mécanismes de rotation. - 1. CALCUL DE LA VARIATION RÉVERSIBLE D'AIMANTATION DUE A LA ROTATION DES VECTEURS $\mathbf{J}_{\mathbf{s}}$; SUSCEPTIBILITÉ INITIALE ROTATIONNELle (Fig. 15). - Considérons un vecteur $\mathbf{J}_{\mathbf{s}}$ initialement suivant un axe de facile aimantation faisant un angle $\psi$ avec la direction d'application du champ $\mathbf{H}$. Sous l'influence du champ $\mathbf{H}, \mathbf{J}_{\mathrm{s}}$ tourne d'un angle $\alpha$ et vient en $\left(\mathbf{J}_{\mathrm{s}}\right)_{1}$. L'axe de facile aimantation est ici un axe quaternaire et le vecteur $\mathbf{J}_{\mathrm{s}}$ reste au
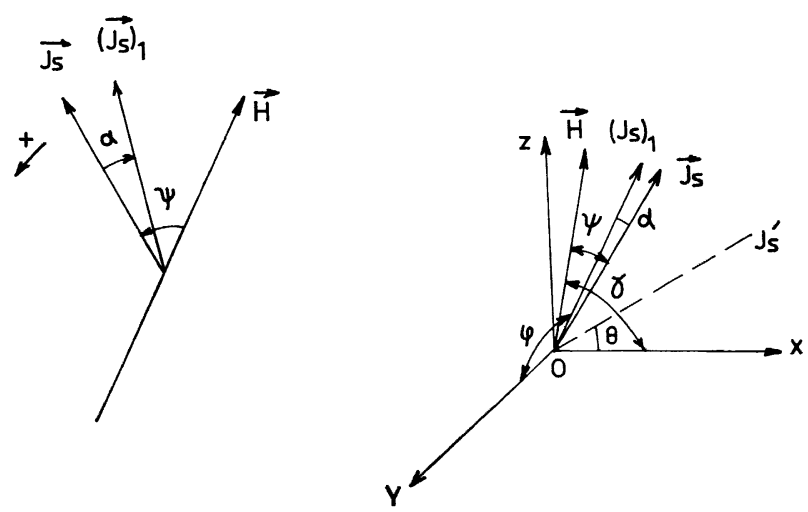

FIG. 15. - Les directions de $\mathbf{H}$ et des vecteurs $\mathbf{J}_{\mathbf{s}}$ et $\mathbf{J}_{\mathbf{s}^{\prime}}$ avant et après application de ce champ. voisinage de celui-ci. Dans ces conditions l'énergie s'écrit :

$$
\begin{aligned}
W & =K_{1} \sin ^{2} \alpha-\mathbf{H} .\left(\mathbf{J}_{\mathrm{s}}\right)_{1} \\
& =K_{1} \sin ^{2} \alpha-H J_{\mathrm{s}} \cos (\alpha+\psi) .
\end{aligned}
$$

Le nouvel équilibre s'obtient pour $\partial W / \partial \alpha=0$ soit :

$$
-H J_{\mathrm{s}}(\sin \psi+\cos \psi \operatorname{tg} \alpha)=2 K_{1} \sin \alpha .
$$

En remarquant que $\alpha$ est petit et en se bornant au premier ordre, il vient :

$$
\alpha=\frac{-X \sin \psi}{1+X \cos \psi} \quad \text { avec } \quad X=\frac{H J_{\mathrm{s}}}{2 K_{1}} .
$$

Comme dans la gamme de champs envisagée dans la mesure $X$ est toujours petit devant $1, \alpha$ s'écrit :

$$
\alpha \simeq-X \sin \psi
$$

La variation d'aimantation consécutive à la rotation $\alpha$ d'un vecteur $\mathbf{J}_{\mathrm{s}}$ s'écrit suivant $\mathbf{H}$ :

$$
\mathrm{d} J=-\alpha J_{\mathrm{s}} \sin \psi
$$

soit, compte tenu de la valeur de $\alpha$ :

$$
\mathrm{d} J=\frac{H J_{\mathrm{s}}^{2}}{2 K_{1}} \sin ^{2} \psi
$$


Avec les notations que nous avons adoptées (Fig. 15) et en exprimant $\sin ^{2} \psi$, il vient :

$$
\mathrm{d} J=\frac{H J_{\mathrm{s}}^{2}}{2 K_{1}}\left[1-\sin ^{2} \varphi \cos ^{2}(\theta-\gamma)\right] .
$$

La variation d'aimantation globale s'obtient en sommant sur l'ensemble des vecteurs $\mathbf{J}_{\mathrm{s}}$ compte tenu de leur répartition.

Dans l'état désaimanté, la répartition des vecteurs $\mathbf{J}_{\mathbf{s}}$ est définie par les probabilités normées $P_{i}^{\prime}\left(\beta_{i}\right), i$ de 1 à 3 . Mais l'application du champ de mesure modifie cette répartition et nous devons en tenir compte dans le calcul de l'aimantation. Nous allons déterminer cette modification. Dans l'état désaimanté nous avons une certaine répartition $P_{i}^{\prime}\left(\beta_{i}\right)$ que l'on peut exprimer en fonction de $\psi$. Après application du champ $\mathbf{H}, \psi$ est devenu $\psi^{\prime}$; il nous faut $P_{i}^{\prime}\left(\psi^{\prime}\right)$. A une variation $\mathrm{d} \psi$ autour de $\psi$ correspond une variation $\mathrm{d} \psi^{\prime}$ autour de $\psi^{\prime}$. On a :

$$
\mathrm{d} \psi^{\prime}=\mathrm{d} \psi\left(1+\frac{\partial \alpha}{\partial \psi}\right)
$$

Exprimons que le nombre de vecteurs $\mathbf{J}_{\mathbf{s}}$ contenu dans l'élément $P_{i}^{\prime}(\psi)$ d $\psi$ est le même que celui contenu dans l'élément $P_{i}^{\prime}\left(\psi^{\prime}\right) \mathrm{d} \psi^{\prime}$. On a alors :

$$
P_{i}^{\prime}\left(\psi^{\prime}\right)=P_{i}^{\prime}(\psi) \frac{\mathrm{d} \psi}{\mathrm{d} \psi^{\prime}}=P_{i}^{\prime}(\psi) \frac{1}{1+\frac{\partial \alpha}{\partial \psi}}
$$

Comme dans la gamme de champs envisagée $X$ est toujours petit devant 1 , il en est de même de

$$
\frac{\partial \alpha}{\partial \psi}=-X \cos \psi
$$

On a finalement :

$$
P_{i}^{\prime}\left(\psi^{\prime}\right)=P_{i}^{\prime}(\psi)\left(1-\frac{\partial \alpha}{\partial \psi}\right)
$$

avec :

$$
\frac{\partial \alpha}{\partial \psi}=-\frac{H J_{\mathrm{s}}}{2 K_{1}} \sin \varphi \cos (\theta-\gamma)
$$

Nous pouvons calculer la variation d'aimantation et la susceptibilité rotationnelle correspondante. Elle a pour expression :

$$
\begin{aligned}
& \chi_{\text {rot }}=\frac{J_{\mathrm{s}}^{2}}{2 K_{1}} \int_{-\pi / 2+\gamma}^{\pi / 2+\gamma} {\left[1-\sin ^{2} \varphi \cos ^{2}(\theta-\gamma)\right] \mathrm{d} \theta } \\
& \times \int_{0}^{\pi / 2} P_{i}^{\prime}(\varphi, \theta)\left[1-\frac{\partial \alpha}{\partial \psi}\right] \sin \varphi \mathrm{d} \varphi .
\end{aligned}
$$

La déformation de la répartition varie avec $H$ quand on aimante la substance et il faut considérer une action intégrée dans la gamme de variation du champ de mesure. Nous désignerons par $\langle H\rangle$ cette fonction de $H$.

Nous allons envisager les susceptibilités rotationnelles relatives aux divers types de répartition.

1.1 Répartition isotrope des aimantations spontanées. - On a alors :

$$
P^{\prime}(\varphi, \theta)=\frac{1}{\pi}
$$

ce qui entraîne une susceptibilité $\left(\chi_{\text {rot }}\right)$ isotrope indépendante de $\gamma$ ayant pour valeur :

$$
\left(\chi_{\text {rot }}\right)_{\text {isotrope }}=\frac{J_{\mathrm{s}}^{2}}{3 K_{1}}+\frac{J_{\mathrm{s}}^{3}}{16 K_{1}^{2}}<H>.
$$

1.2 Répartition anisotrope des aimantations spontanées. - La susceptibilité rotationnelle se compose alors de 4 termes :

$$
\begin{aligned}
& \underset{i \mathrm{de} 1 \mathrm{a} \mathbf{3} 3}{\left(\chi_{\mathrm{rot}}\right)_{i}}=\frac{J_{\mathrm{s}}^{2}}{2 K_{1}}\left\{\int_{-\pi / 2+\gamma}^{\pi / 2+\gamma} \mathrm{d} \theta \int_{0}^{\pi / 2} P_{i}^{\prime}(\varphi, \theta) \sin \varphi \mathrm{d} \varphi-\int_{-\pi / 2+\gamma}^{\pi / 2+\gamma} \cos ^{2}(\theta-\gamma) \mathrm{d} \theta \int_{0}^{\pi / 2} P_{i}^{\prime}(\varphi, \theta) \sin ^{3} \varphi \mathrm{d} \varphi\right\}+ \\
& +\frac{J_{\mathrm{s}}^{3}}{4 K_{1}^{2}}<H>\left\{\int_{-\pi / 2+\gamma}^{\pi / 2+\gamma} \cos (\theta-\gamma) \mathrm{d} \theta \int_{0}^{\pi / 2} P_{i}^{\prime}(\varphi, \theta) \sin ^{2} \varphi \mathrm{d} \varphi-\int_{-\pi / 2+\gamma}^{\pi / 2+\gamma} \cos ^{3}(\theta-\gamma) \mathrm{d} \theta \int_{0}^{\pi / 2} P_{1}^{\prime}(\varphi, \theta) \sin ^{4} \varphi \mathrm{d} \varphi\right\} .
\end{aligned}
$$

Le calcul numérique de ces intégrales ne présente aucune difficulté. Nous avons en principe tous les éléments nous permetta nt de tenir compte de ce premier effet.

2. INFLUENCE DES FAIBLES ROTATIONS SUR LA RÉPARTITION DES VECTEURS POLARISATION ; CALCUL DES VARIATIONS D'AIMANTATION QUI EN RÉSULTENT. Considérons une paroi séparant deux domaines d'aimantation $\mathbf{J}_{1}$ et $\mathbf{J}_{2}$. Sous l'influence du champ $\mathbf{H}$ ces vecteurs tournent respectivement des angles $\alpha_{1}$ et $\alpha_{2}$ et le vecteur polarisation $\mathbf{P}=\mathbf{J}_{2}-\mathbf{J}_{1}$ tourne de l'angle $\omega=\frac{\alpha_{1}+\alpha_{2}}{2}$. Dans le cas d'une paroi à $180^{\circ}$ on montre que $\omega=0$; il n'en est plus de même pour une paroi à $90^{\circ}$. Si $\varphi$ et $\varphi+\pi / 2$ sont les angles que font respectivement $\mathbf{J}_{1}$ et $\mathbf{J}_{2}$ avec $\mathbf{H}, \omega$ s'écrit en se limitant au premier ordre :

$$
\omega=-\frac{1}{2} X(\cos \varphi+\sin \varphi) .
$$

Mais ce qui nous intéresse ce n'est pas la variation de $\omega$ en fonction de la position angulaire de $\mathbf{J}_{\mathbf{s}}$, mais de la position angulaire de la polarisation. $\mathbf{P}$ fait avec le 
champ $\mathbf{H}$ un angle égal à $\varphi+\frac{3 \pi}{4}$. Soit $\psi$ cet angle ; on a $\varphi=\psi-\frac{3 \pi}{4}$. On obtient pour $\omega$ en fonction de $\psi$ :

$$
\omega=\frac{H J_{\mathrm{s}}}{2 \sqrt{2} K_{1}} \cos \psi=\frac{H J_{\mathrm{s}}}{2 \sqrt{2} K_{1}} \sin \varphi \cos (\theta-\gamma)
$$

$\varphi, \theta, \gamma$, sont les angles habituels (cf. Fig. 15) et $\psi$ est l'angle de $\mathbf{P}$ avec $\mathbf{H} . \partial \omega / \partial \psi$ s'écrit :

$$
\begin{aligned}
\frac{\partial \omega}{\partial \psi}=\frac{-H J_{\mathrm{s}}}{2 \sqrt{2} K_{1}}\left\{1-\sin ^{2} \varphi \cos ^{2}(\theta-\gamma)\right\}^{1 / 2} & = \\
& =\frac{-H J_{\mathrm{s}}}{2 \sqrt{2} K_{1}} \sin \psi .
\end{aligned}
$$

La rotation du vecteur $\mathbf{P}$ a lieu dans le plan $\mathbf{P}, \mathbf{H}$; elle entraîne une variation de $\varphi$ et de $\theta$ qui s'écrit au premier ordre :

$$
\begin{gathered}
\delta \varphi=\omega \cdot \frac{\cos \varphi \cos (\theta-\gamma)}{\left\{1-\sin ^{2} \varphi \cos ^{2}(\theta-\gamma)\right\}^{1 / 2}} \\
\delta \theta=\omega\left\{1-\frac{\cos ^{2} \varphi \cos ^{2}(\theta-\gamma)}{1-\sin ^{2} \varphi \cos ^{2}(\theta-\gamma)}\right\}^{1 / 2}
\end{gathered}
$$

avec $\delta \varphi^{2}+\delta \theta^{2}=\omega_{2}$.

Les nouvelles répartitions $P_{k}^{\prime *}(\varphi, \theta)$ s'écrivent :

$P_{k}^{\prime *}(\varphi, \theta)=P_{k}^{\prime}(\varphi, \theta)\left(1-\frac{\partial \omega}{\partial \psi}\right)=$

$=P_{k}^{\prime}(\varphi, \theta)\left(1+\frac{<H>}{2 \sqrt{2} K_{1}}\left\{1-\sin ^{2} \varphi \cos ^{2}(\theta-\gamma)\right\}^{1 / 2}\right)$.

En effet, la déformation de la répartition évolue en fonction du champ magnétisant et il faut considérer une action intégrée de celui-ci que nous désignerons par $\langle H\rangle$.

Les nouvelles lois d'aimantation s'écrivent sans difficulté. Il suffit de remplacer les $Q_{k \mathrm{r}}$ et $Q_{k \mathrm{i}}$ par les nouvelles valeurs :

$$
\begin{aligned}
& \left(Q_{k \mathrm{r}}^{\gamma}\right)^{*}=\int_{-\pi / 2+\gamma}^{\pi / 2+\gamma}\left\{\cos ^{2}(\theta-\gamma)+2 \delta \theta \sin (\theta-\gamma)\right\} \mathrm{d} \theta \int_{0}^{\pi / 2} P_{k}^{\prime *}\left\{\sin ^{3} \varphi+\delta \varphi \sin ^{2} \varphi \cos \varphi\right\} \mathrm{d} \varphi . \\
& \left(Q_{k \mathrm{i}}^{\gamma}\right)^{*}=\int_{-\pi / 2+\gamma}^{\pi / 2+\gamma}\left\{\cos ^{3}(\theta-\gamma)+3 \delta \theta \cos ^{2}(\theta-\gamma) \sin (\theta-\gamma)\right\} \mathrm{d} \theta \int_{0}^{\pi / 2}{P_{k}^{\prime *}}_{k}^{*}\left\{\sin ^{3} \varphi+\delta \varphi \sin ^{2} \varphi \cos \varphi\right\} \mathrm{d} \varphi .
\end{aligned}
$$

Ces nouvelles intégrales se calculent numériquement sans difficulté. 